\title{
Case Study Based Convergence Behaviour Analysis of ACO Applied to Optimal Design of Water Distribution Systems
}

\author{
Aaron C. Zecchin, Holger R. Maier and Angus R. Simpson \\ School of Civil and Environmental Engineering, The University of Adelaide
}

Australia

\section{Introduction}

Based on its analogical link with nature, ant colony optimisation (ACO) aims to determine the least cost solution to an optimisation problem via the process of stigmergy (Dorigo et al. 2000). That is, the colony of artificial ants repeatedly stochastically constructs solutions and utilises the information gained from these solutions to guide the construction of future solutions. This process occurs in an attempt to increase the likelihood of the colony constructing the optimal solution. Each individual ant operates essentially randomly, but through alteration of its environment, a colony learns and assimilates information as a collective.

A conceptualised characteristic of this process is that the colony's searching behaviour changes with time. That is, it undergoes a highly variable, and broad reaching, initial search as the colony learns about the solution space, followed by a subsequent intensified searching

$\mathcal{E}$ in smaller regions of the solution space that the colony has learned as being promising. As

$\underset{0}{0}$ such, ACO can be visualised as an initially widely spread colony converging to a point, or $\stackrel{\Phi}{\subseteq}$ region, within the solution space.

들 Typically algorithms, such as ACO, are assessed only based on their performance in terms O $\Phi$ addition to these performance based indicators, much can be learned about the different algorithms by considering the behaviour of their searching and converging process. Algorithm developers qualitatively discuss mechanisms as being exploration encouraging 3 or exploitation encouraging (Colorni et al. 1996). The question arises as to the actual (f) manifestation of these mechanisms in an algorithm's searching behaviour in terms of 을 measurable quantities.

W Within this chapter, two simple statistics for achieving this are implemented. A statistic is is formulated that describes the topological nature of the spread of solutions through the \&f solution space, termed the mean colony distance. Combining this statistic with a measure of 0 the quality of the solutions being found, it is shown to give significant insight into the $\varangle$ behaviour of selected ACO algorithms as the colonies converge. This chapter presents a ळ purely computational analysis. For a theoretical treatment of ACO, the reader is referred to Oㅇ other work (e.g. Gutjahr, 2002). 
In this chapter, a case study based analysis of the convergence behaviour of four ACO algorithms applied to the water distribution system problem (WDSP) is presented. Water distribution systems are one of the largest and most costly infrastructure in all developed societies. As such, the problem of the optimal design of such systems has been a large area of active research within the Civil Engineering field since the late 1960s. The WDSP represents a difficult, highly constrained combinatorial optimisation problem.

The four ACO algorithms studied are: ant system (AS), the first and most basic form of ACO (Dorigo et al. 1996); the elitist AS ( $\mathrm{AS}_{\text {elite }}$, a version of AS utilising an elitism strategy (Dorigo et al. 1996); the elitist-rank $\mathrm{AS}\left(\mathrm{AS}_{\mathrm{rank}}\right)$, similar to $\mathrm{AS}_{\text {elite }}$ but with a rank based prioritisation of information about the solution space obtained by the ants (Bullnheimer et al. 1999); the max-min AS (MMAS), an ACO algorithm that bounds the evolution of the artificial pheromone trails (Stützle \& Hoos 2000). On a macro level, these algorithms differ in their assimilation of new information with previously learned information. By considering the comparative convergence behaviour of these algorithms, insight into the practical outworking of their different formulations is gained.

The chapter is structured as follows. Firstly, in section 2 ACO is briefly presented and the pheromone updating mechanisms of the four algorithms are outlined. In section 3 , the WDSP is explained and defined. Section 4 presents the application of ACO to the WDSP, where the issues of unconstrained problem transformation and problem graph structure are discussed. In section 5, a topology of the solution space is defined and the topological measure used to quantify the spread of the colony's solutions through the solution space is presented. In section 6, a detailed case study based analysis of the convergence behaviour of the algorithms is undertaken. Finally, the conclusions are given in section 7.

\section{Ant Colony Optimisation Algorithms}

This section is intended to provide a brief overview of ACO for the purpose of representing it in a multi-graph framework, so that its application to the WDSP in section 4 is easier to understand. For a detailed discussion of the traditional formulation, the reader is referred to Dorigo et al. (1999).

ACO is an evolutionary algorithmic optimisation process based on the analogy of a colony of foraging ants determining the shortest path between a food source and its nest (see Dorigo et al. (1996) for examples). The colony is able to optimise the excursions of its ants through the process of stigmergy (Dorigo et al. 2000), where stigmergy refers to the indirect form of communication between the ants that arises from their deposition of pheromone trails. These trails act as sign posts encouraging ants to follow them. Gradually, over time increasingly shorter pheromone trails will be reinforced with greater amounts of pheromone. This in turn will encourage more ants to follow them, potentially finding small improvements, leaving the pheromone on the less frequently used, and longer, paths to evaporate into non-existence.

ACO deals with a combinatorial optimisation problem organised as a graph $\mathcal{G}(N, L)$, where $N$ is the set of nodes and $L$ is the set of edges linking the nodes (the structure of the graph is unique for each problem type). A candidate solution $S$ to the problem is constructed by an ant selecting a feasible path through $\mathcal{G}(N, L)$. The feasibility of the path is ensured by a special constraint function $\Theta$, which lists the edges that are available for selection based on the previously constructed path of the ant. That is, given an ant has constructed a path $S^{\prime}$, 
then $\Theta\left(S^{\prime}\right)$ describes the set of edges available for selection. An ant's tour is complete when $\Theta\left(S^{\prime}\right)=\varnothing$, at which point, $S^{\prime} \in \mathcal{S}$, the set of all feasible tours through the graph.

A probabilistic decision policy is implemented at each sequential node in an ant's path for the selection of a new edge from the set $\Theta$ to add to their partially constructed path $S$. This policy is dependent on the pheromone intensity on a particular edge (representative of the colony's learned information) and the desirability of the edge (a measure of the local effect that the selection of a particular edge will have on the value of the objective function (Dorigo et al. 1996)). More precisely, the probability $p_{j \mid S}(t)$ that edge $j \in \Theta\left(S^{\prime}\right)$ will be selected in iteration $t$ given an ant's partially constructed tour $S^{\prime}$ is

$$
p_{j \mid S^{\prime}}(t)=\frac{\left[\tau_{j}(t)\right]^{\alpha}\left[\eta_{j}\right]^{\beta}}{\sum_{l \in \Theta\left(S^{\prime}\right)}\left[\tau_{l}(t)\right]^{\alpha}\left[\eta_{l}\right]^{\beta}},
$$

where $\tau_{j}(t)$ is the pheromone concentration associated with edge $j$ at iteration $t, \eta_{j}$ is the desirability factor and, $\alpha$ and $\beta$ are the parameters controlling the relative importance of pheromone and desirability, respectively, in the decision process. If $\alpha>>\beta$ the algorithm will make decisions based mainly on the learned information, as represented by the pheromone, and if $\beta>>\alpha$ the algorithm will act as a greedy heuristic selecting mainly the lowest cost options, disregarding the impact of these decisions on the final solution quality. At the end of an iteration, all ants from the colony have constructed feasible paths through $\mathcal{G}(N, l)$. The edge pheromone values $\tau_{j}, j \in L$ are updated to include the new information gained by the colony from the set of the new paths created by the colony $\Sigma(\mathrm{t})=\left\{S_{1}(t), \ldots, S_{m}(t)\right\}$, where $S_{k}(t) \in S$ is the path chosen by ant $k$, and $m$ is the number of ants in the colony. The pheromone is updated from one iteration to the next by the transitional relationship

$$
\tau_{j}(t+1)=\rho \tau_{j}(t)+\Delta \tau_{j}(\Sigma(t), t)
$$

where $\rho \in(0,1)$ is the pheromone persistence factor that mimics the natural operation of pheromone decay, and governs the influence of previously learned information on future decisions, and $\Delta \tau_{j}(\Sigma(t), t)$ is the pheromone addition for edge $j$, which governs the influence of the newly acquired information from iteration $t$, on future decisions. The function $\Delta \tau_{j}(\Sigma(t), t)$ can be viewed as the value placed on edge $j$ based on the information contained in $\Sigma(t)$, where value can be interpreted to mean the likelihood that edge $j$ is contained in $S^{*}$, the optimal solution to the problem. Practically, this means that edge $j \in S$ is considered to have more value than $j^{\prime} \in S^{\prime}$ if $f(S)<f\left(S^{\prime}\right)$. The information in this set is essentially the resulting sample of relationships between the edges of the solutions in $\Sigma(t)$ and the corresponding function values of these solutions. The premise of ACO is that by repeated iteration of this process the colony of ants will collectively guide itself to find the optimal path through $G(N, l)$.

The main differentiating factor between ACO variants is the formulation of $\Delta \tau_{j}(\Sigma(t), t)$, as this describes the manner in which new information is assimilated with existing learned information. In the following subsections, the pheromone updating procedures of the four ACO variants studied in this chapter are described. All of these algorithms use the decision policy from (1). 


\subsection{Ant System (AS)}

Ant System (AS) (Dorigo et al. 1996) is the original and simplest ACO algorithm. For AS, all of the ants within the colony add pheromone to their paths, and as such $\Delta \tau_{j}(t)$ is a function of all the solutions found at iteration $t$ and is given by

$$
\Delta \tau_{j}(t)=\sum_{k=1}^{m} \frac{Q}{f\left(S_{k}(t)\right)} I_{S_{k}(t)}\{j\}
$$

where $m$ is the number of ants in the colony (i.e. the number of solutions generated at each iteration), $Q$ is the pheromone addition factor, $f(\cdot)$ is the objective function to be minimised and $I_{A}\{a\}$ is the indicator function (equal to one if $a \in A$ and zero otherwise). From (3), it is clear that better solutions (i.e. solutions with lower objective $f$ values) are rewarded with greater pheromone addition.

\subsection{Elitist Ant System $\left(\mathrm{AS}_{\text {elite }}\right)$}

To exploit information about the current global-best solution, Dorigo et al. (1996) proposed the use of an algorithm known as Elitist Ant System ( $\left.\mathrm{AS}_{\text {elite }}\right)$. This algorithm uses elitist ants, which only reinforce the path of the current global-best solution after every iteration (analogous to elitism strategies used in genetic algorithms). Thus, the pheromone addition is given by

$$
\Delta \tau_{j}(t)=\sum_{k=1}^{m} \frac{Q}{f\left(S_{k}(t)\right)} I_{S_{k}(t)}\{j\}+\sigma \frac{Q}{f\left(S_{g b}(t)\right)} I_{S_{g b}(t)}\{j\}
$$

where the first part of (4) corresponds to the pheromone addition from the colony, as defined for AS in (3), and the second part corresponds to the pheromone addition from the elitist ants, where $\sigma$ is the number of elitist ants and $S_{g b}(t)$ is the set of edges comprising the global best solution found up until iteration $t$ (i.e. this is equivalent to the addition of pheromone from $\sigma$ ants). The updating rule for $\mathrm{AS}_{\text {elite }}$ allows for exploration, as each of the $m$ solutions found by the colony receives a pheromone addition, but also encourages exploitation, as the global-best path is reinforced with the greatest amount of pheromone. As $\sigma$ increases, so does the emphasis on exploitation.

\subsection{Elitist-Rank Ant System ( AS $\left._{\text {rank }}\right)$}

Proposed by Bullnheimer et al. (1999), the Elitist-Rank Ant System (AS rank $_{\text {) }}$ further develops the idea of elitism used in $\mathrm{AS}_{\text {elite }}$ to involve a rank-based updating scheme. In $\mathrm{AS}_{\text {rank, }} \sigma$ elitist ants reinforce the current global-best path, as in $\mathrm{AS}_{\text {elite, }}$ and the ants that found the top $\sigma-1$ solutions within the iteration add pheromone to their paths with a scaling factor related to the rank of their solution. The pheromone update formula for $\mathrm{AS}_{\mathrm{rank}}$ is given by

$$
\Delta \tau_{j}(t)=\sigma \frac{Q}{f\left(S_{g b}(t)\right)} I_{S_{g b}(t)}\{j\}+\sum_{k=1}^{\sigma-1}(\sigma-k) \frac{Q}{f\left(S_{(k)}(t)\right)} I_{S_{(k)}(t)}\{j\}^{\prime}
$$

where the first part of (5) corresponds to the addition from the elitist ants, and the second part from the ranked ants, where $S_{(k)}(t)$ is the set of edges selected by the $k^{\text {th }}$ ranking ant in iteration $t$. The edges that are selected by the $k^{\text {th }}$ ranking ant receive pheromone equivalent 
to the addition from $(\sigma-k)$ ants. The potential advantages of this formulation, compared with AS and $\mathrm{AS}_{\text {elite, }}$ are (i) only the top $\sigma-1$ ranked ants are used to lay pheromone (and not all $m$ ants), which allows for the retention of only good information, and (ii) greater importance is given to the higher ranking ants (i.e. the top ranked solution receives $\sigma-1$ times the normal amount of pheromone and the $(\sigma-1)^{\text {th }}$ ranked solution receives only the normal pheromone amount), so that better edges receive more pheromone.

\subsection{Max-Min Ant System (MMAS)}

To overcome the problem of premature convergence whilst still allowing for exploitation, Stützle and Hoos (2000) developed the Max-Min Ant System (MMAS). The basis of MMAS is the provision of dynamically evolving bounds on the pheromone trail intensities such that the pheromone intensity on all paths is always within a specified lower bound $\tau_{\min }(t)$ of a theoretically asymptotic upper limit $\tau_{\max }(t)$, that is $\tau_{\min }(t) \leq \tau_{j}(t) \leq \tau_{\max }(t)$ for all edges $j$. As a result of stopping the pheromone trails from decaying to zero, all paths always have a nontrivial probability of being selected, and thus wider exploration of the search space is encouraged. The pheromone bounds at iteration $t$ are given by (Stützle \& Hoos 2000)

$$
\tau_{\min }(t)=\frac{\tau_{\max }(t)\left(1-\sqrt[n]{P_{\text {best }}}\right)}{\left(N O_{\text {avg }}-1\right) \sqrt[n]{P_{\text {best }}}}, \tau_{\text {max }}(t)=\frac{1}{1-\rho} \frac{Q}{f\left(S^{g b}(t)\right)}
$$

where $P_{\text {best }}$ is the (user selected) probability that the current global-best path, $S_{g b}(t)$, will be selected, given that all non-global best edges have a pheromone level of $\tau_{\min }(t)$ and all globalbest edges have a pheromone level of $\tau_{\max }(t)$, and $N O_{\text {avg }}$ is the average number of edges at each decision point. It should be noted that lower values of $P_{\text {best }}$ indicate tighter bounds.

As the bounds serve to encourage exploration, MMAS adds pheromone only to the iteration-best ant's path $S_{(1)}(t)$ at the end of an iteration in order to ensure the exploitation of good solutions. To further exploit good information, the global-best solution $S_{g b}(t)$ is updated every $T_{g b}$ iterations. The MMAS pheromone update is given by

$$
\Delta \tau_{j}(t)=\frac{Q}{f\left(S_{(1)}(t)\right)} I_{S_{(1)}(t)}\{j\}+\frac{Q}{f\left(S_{g b}(t)\right)} I_{S_{g b}(t)}\{j\} \cdot I_{\mathrm{N}}\left\{t / T_{g b}\right\}
$$

where the first part of (7) corresponds to the addition from the iteration best ant, and the second part from the global best ant, where $\mathrm{N}$ is the set of natural numbers.

MMAS also utilises another mechanism known as pheromone trail smoothing (PTS). This reduces the relative difference between the pheromone intensities, and further encourages exploration. The PTS operation is given by

$$
\tau_{j}(t) \leftarrow \tau_{j}(t)+\delta\left(\tau_{\max }(t)-\tau_{j}(t)\right),
$$

where $0 \leq \delta \leq 1$ is the PTS coefficient. If $\delta=0$, PTS has no effect, whereas if $\delta=1$, all pheromone paths are scaled up to $\tau_{\max }(t)$. The pheromone updating process of MMAS can be summarised as the three step process of: (i) decay and addition by (2) and (7), (ii) bounding by (6), and (iii) smoothing by (8). 


\section{The Water Distribution System Optimisation Problem}

Water distribution systems (WDSs) consist of the system of pipes, pumps, valves etc. that delivers water from sources to consumers. From an optimisation perspective, the water distribution system problem (WDSP) is defined as the selection of the lowest cost combination of appropriate component sizes (e.g. pipes) and settings (e.g. valve settings) such that the criteria of water demands and other design constraints (e.g. minimum pressures) are satisfied. A simple example of this is as follows. Consider two networks, the first comprising pipes with small diameters and the second comprising pipes with large diameters. The first network has a low cost, but as the pipe diameters are small, the frictional pressure loss through the network will be greater, which is likely to result in insufficient pressure at the demand points (nodes). The second system is likely to provide more than adequate pressure, as the pipe diameters are large, but is also more expensive. The optimal design is the least cost combination of pipe sizes that are able to provide adequate pressure at each of the nodes. Within the WDSP, the decision variables are associated with the pipes within the system where, more specifically, the design options are the following, (i) a diameter for a new pipe, (ii) the cleaning of an existing pipe to reduce the hydraulic resistance, and (iii) no action.

As outlined in Zecchin et al. (2005), for the WDSP, a design involves the selection of a series of design options for all or some of the pipes within the network. A WDS design $\Omega=\left\{\Omega_{1}, \ldots, \Omega_{n}\right\}$ is defined as a set of $n$ decisions where $n$ is the number of pipes to be sized and/or rehabilitated, and $\Omega_{i}$ is the selected option for pipe $i$, and is defined as $\Omega_{i} \in \Lambda_{i}=\left\{\omega_{i, 1}, \ldots, \omega_{i, N O}\right\}$, where $\Lambda_{i}$ is the set of all $N O_{i}$ options available for pipe $i$. For each option there is an associated cost, $c\left(\Omega_{i}\right)$, of implementing that option, and one of three actions as listed above.

The constraints on a solution are categorized as design constraints and hydraulic constraints. A design constraint is an inequality constraint that defines the minimum acceptable performance of a design, whereas hydraulic constraints are equality constraints that describe the distribution of the flow of water through the WDS (based on the fundamental equations for fluid flow within a closed conduit and the governing equations for fluid flow within a looped network). The design constraint for the WDSP specifies the minimum allowable pressure at each node, and is given as

$$
H_{i, j} \geq \underline{H}_{i, j} \quad \forall i=1, . ., N_{\text {node }} \quad \forall j=1, . ., N_{\text {pattern }},
$$

where $H_{i, j}$ is the actual head at node $i$ for demand pattern $j, \underline{H}_{i, j}$ is the minimum allowable head at node $i$ for demand pattern $j, N_{\text {node }}$ is the total number of nodes and $N_{\text {pattern }}$ is the number of demand patterns.

The hydraulic equations for fluid flow within a WDS are the conservation of mass and the pipe headloss equations. As the fluid is assumed to be incompressible, the conservation of mass equations dictate that the flow rate into a node is equal to the flow rate out of a node. This can be expressed as

$$
\underline{Q}_{i, j}+\sum_{k \in \Theta_{u, i}} Q_{k, j}-\sum_{k \in \Theta_{d, i}} Q_{k, j}=0 \quad \forall i=1, . ., N_{\text {node }} \quad \forall j=1, . ., N_{\text {pattern }},
$$


where $Q_{i, j}$ is the demand for node $i$ and demand pattern $j$ (by definition, a positive demand is one that draws water from the node), $Q_{k, j}$ is the flow in pipe $k$ for demand pattern $j, \Theta_{u, j}$ is the set of all pipes for which node $i$ is the upstream node, and $\Theta_{d, j}$ is the set of pipes for which node $i$ is the downstream node (note that the sign convention is that positive pipe flow occurs from upstream to downstream).

The headloss equation is written as (Streeter \& Wylie 1997)

$$
H_{i_{u}, j}-H_{i_{d}, j}=r_{\Omega_{i}}\left|Q_{i, j}\right| Q_{i, j}{ }^{a-1} \quad i=1, \ldots, N_{\text {pipe }}, j=1, . ., N_{\text {pattern }},
$$

where $r_{\Omega_{i}}$ is a hydraulic resistance term associated with decision $\Omega_{i}, a$ is the flow exponent, and $N_{\text {pipe }}$ is the number of pipes, including new pipes. The headloss equation used within most WDSPs is the Hazen-Williams equation, for which $r_{\Omega_{i}}$ is expressed as

$$
r_{\Omega_{i}}=A C_{\Omega_{i}}^{-a} D_{\Omega_{i}}^{-b} L_{i} \quad \forall i=1, . ., N_{\text {pipe }} \quad \forall j=1, . ., N_{\text {pattern }}
$$

where $L_{i}$ is the length of pipe $i, D_{\Omega_{i}}$ is the diameter of pipe $i$ for design $\Omega, C_{\Omega_{i}}$ is the HazenWilliams coefficient for pipe $i$ for design $\Omega, A$ is a constant that is dependent on the units used, and $a$ and $b$ are regression coefficients. The adopted values of $A, a$, and $b$ are those used in the hydraulic solver software EPANET2 (Rossman 2000).

The objective is the minimization of the material and installation costs, and so the WDSP can be expressed as

$$
\min C(\Omega)=\sum_{i=1}^{n} c\left(\Omega_{i}\right), \text { Subject to }(9)-(11)
$$

where $C(\Omega)$ is the cost of design $\Omega$ and $c\left(\Omega_{i}\right)$ is the cost of decision $\Omega_{i}$. As is seen from (13), despite the simplicity of the objective function, the complexity of the optimisation problem arises from the nonlinear nature of the constraints dependency on the design options $\Omega_{i}$.

\section{Application of Ant Colony Optimisation to Water Distribution System Optimisation}

\subsection{Transformation of constrained problem}

The WDSP is a constrained optimisation problem. ACO is unable to deal directly with constrained optimisation problems as, within its solution generation, it cannot adhere to constraints that separate feasible regions of a search space from infeasible regions (here feasibility refers to constraints (9)-(11) and not the $\Theta$ function). The standard technique to convert constrained problems to unconstrained problems is to use a penalty function (Coello Coello 2002). ACO algorithms direct their search solely based on information provided by the objective function. To guide the search away from the infeasible region and towards the feasible region, a penalty function increases the cost of infeasible solutions such that they are considered to be poor quality solutions. The unconstrained optimisation problem for the WDSP takes the form of minimising the sum of the real cost plus the penalty cost, that is

$$
\min N C(\Omega)=C(\Omega)+P C(\Omega)
$$


where $N C(\Omega)$ is the network cost for design $\Omega, C(\Omega)$ is the material and installation cost given by (13) and $\operatorname{PC}(\Omega)$ is the penalty cost incurred by $\Omega$. When evaluating a potential design, the set of heads $\left\{H_{i, j}: i=1, \ldots, N_{\text {node }}, j=1, \ldots, N_{\text {pattern }}\right\}$ is calculated by a hydraulic solver. Therefore (10)-(11) are automatically satisfied, and hence, only (9) is required to be considered in the penalty cost. Within this study, $P C(\Omega)$ was taken to be proportional to the maximum nodal pressure deficit induced by $\Omega$ as in Maier et al. (2003). That is

$$
P C(\Omega)=\left\{\begin{array}{cc}
0 & \text { if } H_{i, j} \geq \underline{H}_{i, j}, i=1, \ldots, N_{\text {node }}, j=1, \ldots, N_{\text {pattern }} \\
\left.\max _{(i, j) \in\left[1, N_{\text {node }} \times \times\left[1, N_{\text {pattem }}\right]\right.}\left\{\underline{H}_{i, j}-H_{i, j}\right\}\right) \cdot P E N & \text { otherwise }
\end{array}\right.
$$

where $P E N$ is the penalty factor (user defined) with units of dollars per meter of pressure violation.

\subsection{Modification of ACO elements}

As in used in previous studies (Maier et al. 2003; Zecchin et. al. 2005; Zecchin et. al. 2006; Zecchin et. al. 2007), but formalised here, the graph $\mathcal{G}(N, L)$ of the WDSP can be represented as a multi-graph, with the set of nodes $N=\{1,2, \ldots, n+1\}$. Each node $i \leq n$ is connected to the next via a set of directed edges $\theta_{i}=\left\{(i, i+1)_{j}: j=1,2, \ldots, N O_{i}\right\}$, where $(i, i+1)_{j}$ is the $j^{\text {th }}$ edge connecting node $i$ to node $i+1, N O_{i}$ is the number of edges connecting node $i$ to node $i+1$ and the set of all edges is $l=\left\{s: s \in \theta_{1} \cup \ldots \cup \theta_{n}\right\}$. The edge set $\theta_{i}$ is associated with the set of design options $\Lambda_{i}$, and the edge $(i, i+1)_{j}$ is associated with option $\omega_{i, j}$. A solution $S$, associated with design $\Omega$, is a feasible tour through the graph and is an element of the solution space $S=\left\{S: S=\left\{s_{1}, \ldots, s_{n}\right\}, s_{i} \in \theta_{i}, i=1, \ldots, n\right\}$, where the constraint function $\Theta$ is given by $\Theta\left(\left\{s_{1}, \ldots, s_{i}\right\}\right)=\theta_{i}$ for $i \leq n$.

As the objective is to minimise cost, lower cost options are more desirable. Therefore the desirability of an option is taken as the inverse of the cost of implementing that option (Maier et al. 2003). In other words

$$
\eta_{(i, i+1)_{j}}=1 / c\left(\omega_{i, j}\right)
$$

As lower cost diameter options are more desirable, a bias in the probability towards the selection of lower cost diameters results. For options with zero cost (i.e. the null option), a virtual-zero-cost was selected.

\subsection{Parameter Settings}

One of the limitations of ACO is that an extensive calibration phase is required to determine appropriate parameter settings. From an extensive analysis of ACO applied to the WDSP, Zecchin et al. (2005) determined a series of parameter guidelines relating the five fundamental ACO parameters $\left(\alpha, \beta, \rho, Q, \tau_{0}\right.$, and $m$ ) to WDSP characteristics (such as the number of decision points $n$, the average number of options per decision $N O_{\text {avg }}$, and the cost of key design configurations such as $\Omega^{\max }$, the maximum cost design, and $\Omega^{*}$, the optimum, or near optimum, design). These are summarised in Table 1.

Contrary to other problem types (Dorigo \& Gambardella 1997), Zecchin et al. (2005) found that, for the WDSP, better performance was achieved when the ants gave greater emphasis to the learned pheromone values $\tau$ as opposed to the visibility values $\eta$, as manifested 
through $\alpha>\beta$. Better performance was achieved when the pheromone persistence factor was relatively high, facilitating slow convergence and long memory times for learned information. Zecchin et al. (2005) showed that the ratio of $Q$ to $\tau_{0}$ is important (not the actual values of each) and empirical guidelines were determined accordingly. The best number of ants $m$ was also found to be dependent on the number of options per decision, not just the number of decisions, as for other problem types (Dorigo et al. 1996).

\begin{tabular}{|c|c|}
\hline Parameter & Heuristic \\
\hline$\alpha$ & 1.0 \\
\hline$\beta$ & 0.5 \\
\hline$\rho$ & 0.98 \\
\hline$Q$ & $C\left(\Omega^{\text {max }}\right)$ \\
\hline$\tau_{0}$ & $Q \sqrt{n N O_{\text {avg }}} / N C\left(\Omega^{*}\right)$ \\
\hline$M$ & $n \sqrt{N O_{\text {avg }}}$ \\
\hline
\end{tabular}

Table 1. Parameter guidelines for ACO parameters from Zecchin et al. (2005)

In addition to the guidelines derived for the ACO parameters, the following semideterministic expression for PEN was derived in Zecchin et al. (2005)

$$
P E N=\left|C\left(\Omega^{\max }\right)-C\left(\Omega^{\min }\right)\right| / \Delta H
$$

where $\Omega^{\text {min }}$ is the minimum cost network design, and $\Delta H$ is a user selected pressure deficit, based on the maximum acceptable pressure deficit for a feasible solution as defined by (9). The value of $P E N$ ensures that all networks with a pressure violation greater than or equal to $\Delta H$ (an extremely small value) are made more expensive than the maximum feasible network $\operatorname{cost} C\left(\Omega^{\mathrm{min}}\right)$.

\section{Analysis of Algorithm Convergence Behaviour}

The standard approach to the analysis of optimisation algorithms is to assess their performance on a particular problem from statistics based on the lowest cost achieved by the algorithm (termed best-cost) and the computational time required for the algorithm to find the associated solution (termed search-time). A richer understanding of the performance of an algorithm can be achieved by considering statistics from the solutions generated by the algorithms during their run-time. A typical approach used by many authors (Simpson et al., 1994; Cunha \& Ribeiro, 2004; Afshar \& Marino, 2007) is to track the minimum cost generated in each iteration as a means of assessing the algorithm's convergence behaviour. This statistic is important, as it indicates the effectiveness of the search, but acts only as a surrogate measure of the actual convergence behaviour of the algorithm.

This work aims to extend this qualitative performance assessment to include a topologically based statistic to describe an algorithm's convergence behaviour. From the perspective of $\mathrm{ACO}$, convergence is defined as the point in time at which all ants select the same path 
through the problem graph (i.e. the colony's population of solutions is fixed at a certain point in the solution space $S$ ) from that point onward. Thus, convergence behaviour is the nature of the colony's solution generation up until the point of convergence. Topologically, convergence means that the distance between all solutions generated by the colony is zero. Conversely, a non-converged search will have some spread of the solutions throughout the solution space. It is the quantification and tracking of this spread that is of interest in describing an algorithm's convergence behaviour.

The motive behind convergence analysis is to gain a greater understanding of how the different explorative and exploitative mechanisms in the ACO algorithms considered actually impact the algorithm's search. Below, the topology of the solution space is first defined, and then the adopted convergence metric, the mean colony distance, is presented.

It is important to note that the use of metrics is widely used in evolutionary algorithm based multi-objective optimisation (Deb 2001). However, this is fundamentally different to what is considered here. In multi-objective optimisation, the distribution of solutions throughout the multi-dimensional objective space is of primary interest, and thus the metrics operate in this space. Conversely, this chapter is concerned with the distribution of solutions within the solution space, and, as such, the mean colony distance is defined on this space.

\subsection{Topology of the Solution Space}

Fundamental to any topologically based statistic is the notion of distance between points (solutions) in the solution space. A measure of distance for all elements within the set $S$ is equivalent to defining a metric $d: S \times S \rightarrow R+$ associated with $S$ that defines the distance between two elements $S, S^{\prime} \in \mathcal{S}$ (Cohen 2003). For sets whose elements have no specific numerical relation, the Hamming distance is a natural metric. This has been used by Bose et al. (1994) and Stützle \& Hoos (2000) for the travelling salesperson problem. A generalisation that applies to sets whose elements are equal length lists of objects is

$$
d\left(S, S^{\prime}\right)=\sum_{i=1}^{n} d_{i}\left(s_{i}, s_{i}^{\prime}\right)^{\prime}
$$

where $S=\left\{s_{1}, \ldots, s_{n}\right\}, S^{\prime}=\left\{s_{1}{ }^{\prime}, \ldots, s_{n}{ }^{\prime}\right\}, s_{i}, s_{i}{ }^{\prime} \in \theta_{i}$ and $d_{i}: \theta_{i} \times \theta_{i} \rightarrow \mathrm{R}_{+}$is itself a metric for the set of all possible $i^{\text {th }}$ elements in the list. For the Hamming distance, $d_{i}(\cdot, \cdot)$ is either zero or one, depending whether $s_{i}$ and $s_{i}^{\prime}$ are equal or not. However, if the elements in the set have some other attribute that can be exploited, such as a meaningful ordering based on some property, then the metric can be defined so as to include this information.

Considering (12), it is seen that the selection of an option $\Omega_{i}$ is essentially equivalent to selecting a resistance parameter $r_{\Omega_{i}}$. Therefore, it is meaningful to say that an option is closer to one option than another based purely on the relative differences between their associated resistance parameter values. The list of options $\Lambda_{i}$ for pipe $i$ can therefore be meaningfully ordered by the magnitude of their associated resistance parameter. That is, consider the following ordering of $\Lambda_{i}$, based on the resistance parameter $\underline{\Lambda}_{i}=\left\{\underline{\omega}_{i, 1}, \ldots, \underline{\omega}_{i, N O}\right\}$, where $r_{\underline{\omega}_{i, 1}} \leq \ldots \leq r_{\underline{\omega}_{i, N o},}$, and the distance $d_{i}$ between any two of these options $\underline{\omega}_{i, j}$ and $\underline{\omega}_{i, k}$, is given by

$$
d_{i}\left(\underline{\omega}_{i, j}, \underline{\omega}_{i, k}\right)=|j-k| \text { where } \underline{\omega}_{i, j}, \underline{\omega}_{i, k} \in \Lambda_{i} .
$$


In this context, the distance between two options is the number of positions in the ordered list $\underline{\Lambda}_{i}$ that separates the two options.

\subsection{Mean colony distance}

By ascribing a topology to the search space, the colony of solutions generated within an iteration can be considered to be spread, in some manner, over the topology. This spread of solutions gives an indication of how widely, or tightly, an algorithm is searching. To use the terminology of Colorni et al. (1996), whether the algorithm is exploring broadly through the search space or exploiting smaller regions of the search space. In order to quantify this spread, the mean of the distances between each of the ants' solutions has been used in this chapter, which is henceforth referred to as the mean colony distance $d_{\Sigma}$. Mathematically this is given as the summation of the distances of each unique pair of solutions divided by the total number of pairs, and is expressed as the map $d_{\Sigma}: S^{m} \rightarrow R+$ where

$$
d_{\Sigma}(t)=\frac{2}{m(m-1)} \sum_{k=1}^{m-1} \sum_{l=k+1}^{m} d\left(S_{k}(t), S_{l}(t)\right),
$$

where $m(m-1) / 2$ is the number of unique pairs that exist in a colony of $m$ ants. The usefulness of $d_{\Sigma}$ as a behavioural analysis measure is fully realised when considering its variation with time. For example: periods of high exploration when solutions are spread broadly throughout the search space correspond to large values of $d_{\Sigma}$; periods during which the algorithm converges correspond to a series of decreasing $d_{\Sigma}$ values; the point at which the algorithm converges is given by $d_{\Sigma}=0$, as this indicates that all solutions in $\Sigma(t)$ are equal. As such, $d_{\Sigma}$ provides a direct measure of an algorithm's convergence behaviour.

\section{Case Studies}

Experiments were performed on four different case studies, the Two Reservoir Problem (TRP), the New York Tunnels Problem (NYTP), the Hanoi Problem (HP) and the Doubled New York Tunnels Problem (2-NYTP). The ACO algorithms were coded in FORTRAN 90 with EPANET2 (Rossman 2000) as the hydraulic solver. Parameter settings from Zecchin et al. (2005), summarised in Table 1, were used for parameters $\alpha, \beta, \rho, \tau_{0}, m$, and $Q$ for all algorithms with the adjustment that $\tau_{0}$ was scaled by $\sigma$ for $\mathrm{AS}_{\text {elite }}$ and $\mathrm{AS}_{\text {rank }}$ (in accordance with the logic of the derivation of $Q$ in Zecchin et al. (2005)) and for MMAS, $\tau_{0}$ was set to an arbitrarily high number, as proposed by Stützle \& Hoos (2000). For $\mathrm{AS}_{\text {elite }}$ and $\mathrm{AS}_{\text {rank, }} \sigma$ required calibration for each case study. For MMAS, $f_{\text {global }}$ was set to 10, as in Stützle \& Hoos (2000) and $P_{\text {best }}$ and $\delta$ were calibrated for each case study. The best-cost and search-time statistics for AS, $\mathrm{AS}_{\text {elite, }}$ and $\mathrm{AS}_{\text {rank }}$ and MMAS are as presented in Zecchin et al. (2007).

\subsection{Case Study 1: Two-Reservoirs Problem}

\subsubsection{Preliminaries}

The TRP was initially studied by Gessler (1985), and Simpson et al. (1994) introduced the metric version. The TRP is a 14-pipe network with two reservoirs (Figure 1). The TRP involves three demand cases: a peak hour demand case and two fire loading demand cases. There are nine existing pipes, of which three are considered for rehabilitation, duplication with one of eight pipe sizes, or to be left alone. There are five new pipes that must be sized 
with one of eight diameters. The reader is referred to Simpson et al. (1994) for case study details. The problem, consists of $32,768,000$ possible combinations.

\subsubsection{Results}

Based on the heuristics given in Table $1,\left\{\tau_{0}, m\right\}=\{27,25\}$ and preliminary testing showed that a maximum number of iterations of $I_{\max }=400$ was sufficient for the algorithms to not significantly improve on their solution quality after this point. For each algorithm, a single run for the TRP consisted of 10,000 function evaluations. The range of parameters tested was: $\sigma \in[2,20]$ for $\mathrm{AS}_{\text {elite; }} \sigma \in[2,20]$ for $\mathrm{AS}_{\text {rank; }}\left\{P_{\text {best }}, \delta\right\} \in\left[1 \times 10^{-5}, 0.99\right] \times[0,0.99]$ for MMAS. AS elite achieved a mean performance within $1 \%$ of the known optimum for most of the tested values of $\sigma$, with better performances observed using $3 \leq \sigma \leq 5$. Similarly, $\mathrm{AS}_{\text {rank }}$ achieved a mean performance within $1 \%$ of the known optimum for all tested values of $\sigma>2$ with lower mean best-cost values occurring for $10 \leq \sigma \leq 14$. AS $_{\text {rank }}$ tended to be less sensitive to variations in $\sigma$ than $\mathrm{AS}_{\text {elite, }}$ as it was able to find the optimum in each run for a broader range of values for this parameter. MMAS achieved a mean performance within $1 \%$ of the optimum for values of $P_{\text {best }} \geq 0.001$ and $\delta \leq 0.001$, with the solution quality deteriorating for parameter values outside these ranges. The optimal parameter values were as follows: for $\mathrm{AS}_{\text {elite, }} \sigma=4$; for $\mathrm{AS}_{\text {rank, }} \sigma=10$; for MMAS, $\left\{P_{\text {best }}, \delta\right\}=\left\{0.5,10^{-6}\right\}$.

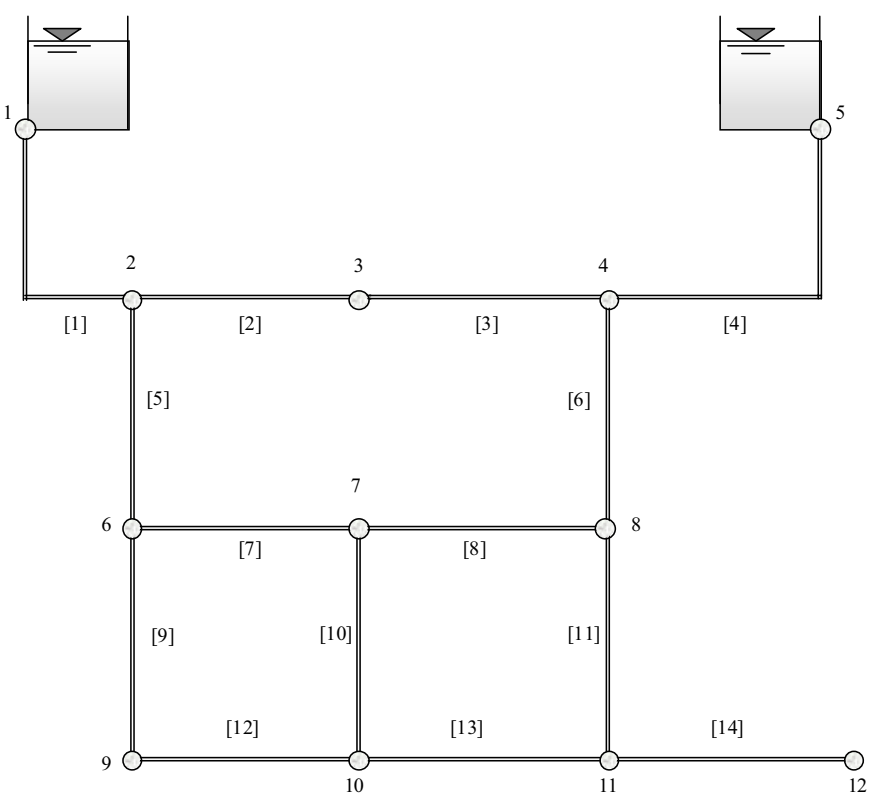

Figure 1. Network layout for the Two-Reservoir Problem

Table 2 gives a comparison of the results obtained using the ACO algorithms considered and those obtained from a selection of other best performing algorithms that have been applied to the discrete version of the TRP previously. A detailed statistical analysis of these algorithms was given in Zecchin et al. (2007), but it is clear that all algorithms performed extremely well (finding the optimum for all 20 runs) and were, comparatively, computationally efficient. 
Plots of the iteration best-costs $f_{\min }(t)$ and the mean colony distance $d_{\Sigma}(t)$, averaged over 20 runs, are given in Figure 2 (a) and (b). In addition to this, other run-time properties (to be discussed) are given in Figure 2 (c). With regard to $f_{\text {min }}(t)$, three distinct phases are observed. The first part of the search, phase-I, is a relatively short phase in which all algorithms find relatively poor quality solutions, which is followed by the second phase, phase-II, in which a dramatic increase in solution quality (reduction in the minimum cost) takes place, which leads into the third phase, phase-III, in which the rate of increase of the solution quality plateaus and the algorithms seem to not find any better solutions (or in some cases, the optimum is found repeatedly).

\begin{tabular}{|c|c|c|c|c|c|c|c|}
\hline \multirow{3}{*}{$\begin{array}{c}\text { Algorithm } \\
\text { AS }\end{array}$} & \multicolumn{6}{|c|}{ Best-cost (\$M) (\% deviation from optimum) } & \multirow{3}{*}{$\begin{array}{c}\begin{array}{c}\text { Mean search-time } \\
\text { (evaluation no.) }\end{array} \\
2,084\end{array}$} \\
\hline & \multicolumn{2}{|c|}{ Minimum } & \multicolumn{2}{|c|}{ Mean } & \multicolumn{2}{|c|}{ Maximum } & \\
\hline & 1.750 & $(0.00)$ & 1.750 & $(0.00)$ & 1.750 & $(0.00)$ & \\
\hline $\mathrm{AS}_{\text {elite }}$ & 1.750 & $(0.00)$ & 1.750 & $(0.00)$ & 1.750 & $(0.00)$ & 1,842 \\
\hline $\mathrm{AS}_{\text {rank }}$ & 1.750 & $(0.00)$ & 1.750 & $(0.00)$ & 1.750 & $(0.00)$ & 1,523 \\
\hline MMAS & 1.750 & $(0.00)$ & 1.750 & $(0.00)$ & 1.750 & $(0.00)$ & 2,993 \\
\hline $\mathrm{PE}^{\mathrm{a}}$ & 1.834 & $(4.80)$ & \multicolumn{2}{|c|}{-} & & 900 \\
\hline $\mathrm{GA}_{\text {prop }}{ }^{\mathrm{b}}$ & 1.750 & $(0.00)$ & 1.759 & $(0.51)$ & 1.812 & (3.54) & 23,625 \\
\hline $\mathrm{GA}_{\text {tout }}{ }^{\mathrm{c}}$ & 1.750 & $(0.00)$ & 1.750 & $(0.00)$ & 1.750 & $(0.00)$ & 8,700 \\
\hline $\mathrm{SA}^{\mathrm{d}}$ & 1.750 & $(0.00)$ & \multicolumn{2}{|c|}{ NA } & \multicolumn{2}{|c|}{ NA } & NA \\
\hline $\mathrm{ACOA}^{\mathrm{e}}$ & 1.750 & $(0.00)$ & 1.769 & (1.09) & 1.813 & $(3.60)$ & 12,455 \\
\hline $\mathrm{TS}^{\mathrm{f}}$ & $1.728^{\mathrm{i}}$ & - & \multicolumn{2}{|c|}{ NA } & \multicolumn{2}{|c|}{ NA } & $\sim 10,000$ \\
\hline $\mathrm{ACOA}_{\text {i-best }} \mathrm{g}$ & 1.750 & $(0.00)$ & 1.750 & $(0.00)$ & 1.750 & $(0.00)$ & 8,509 \\
\hline ACSh & 1.750 & $(0.00)$ & 1.770 & (1.13) & 1.904 & $(8.81)$ & 5,014 \\
\hline \multicolumn{8}{|c|}{$\begin{array}{l}\text { a Partial enumeration (Gessler 1985). }{ }^{\mathrm{b}} \text { GA based on a proportionate selection rule (Simpson et al. 1994). } \\
\text { c Tournament selection GA (Simpson \& Goldberg 1994). }{ }^{\mathrm{d}} \text { Simulated Annealing (Sousa \& Cunha 1999). } \\
\text { e An AS variant that subtracts pheromone (Maier et al. 2003). }{ }^{\mathrm{f}} \text { Tabu Search (Cunha \& Ribeiro 2004). } \\
\text { g Iteration-best updating version of ACOA (Maier et al. 2003). }{ }^{\mathrm{h}} \text { Ant Colony System (Zecchin et. al 2007). } \\
{ }^{i} \text { Not feasible by complete enumeration results (Simpson } \text { et al. 1994). }\end{array}$} \\
\hline
\end{tabular}

Table 2. Comparison of performance of AS, $\mathrm{AS}_{\text {elite, }} \mathrm{AS}_{\mathrm{rank}}, \mathrm{MMAS}$, and other algorithms from the literature applied to the Two-Reservoir Problem. Results for AS, $\mathrm{AS}_{\text {elite, }} \mathrm{AS}_{\text {rank, }}$ and MMAS are based on 20 runs. NA means that the information is not available

These three phases can also be seen clearly when considering the behaviour of $d_{\Sigma}$ in Figure 2 (b). To make the distinction between the phases clearer, the bar chart in Figure 2 (c) indicates when the algorithms are in each of the phases (dark grey for phase-I, light grey for phase-II and the remaining white space for phase-III). For $d_{\Sigma}$, phase-I corresponds to a brief period of extremely broad searching where almost no convergence behaviour is displayed, followed by 
phase-II, in which relatively rapid convergence is observed, and phase-III, in which the rate of convergence either plateaus or decreases gradually to $d_{\Sigma}(t)=0$, the point of convergence.

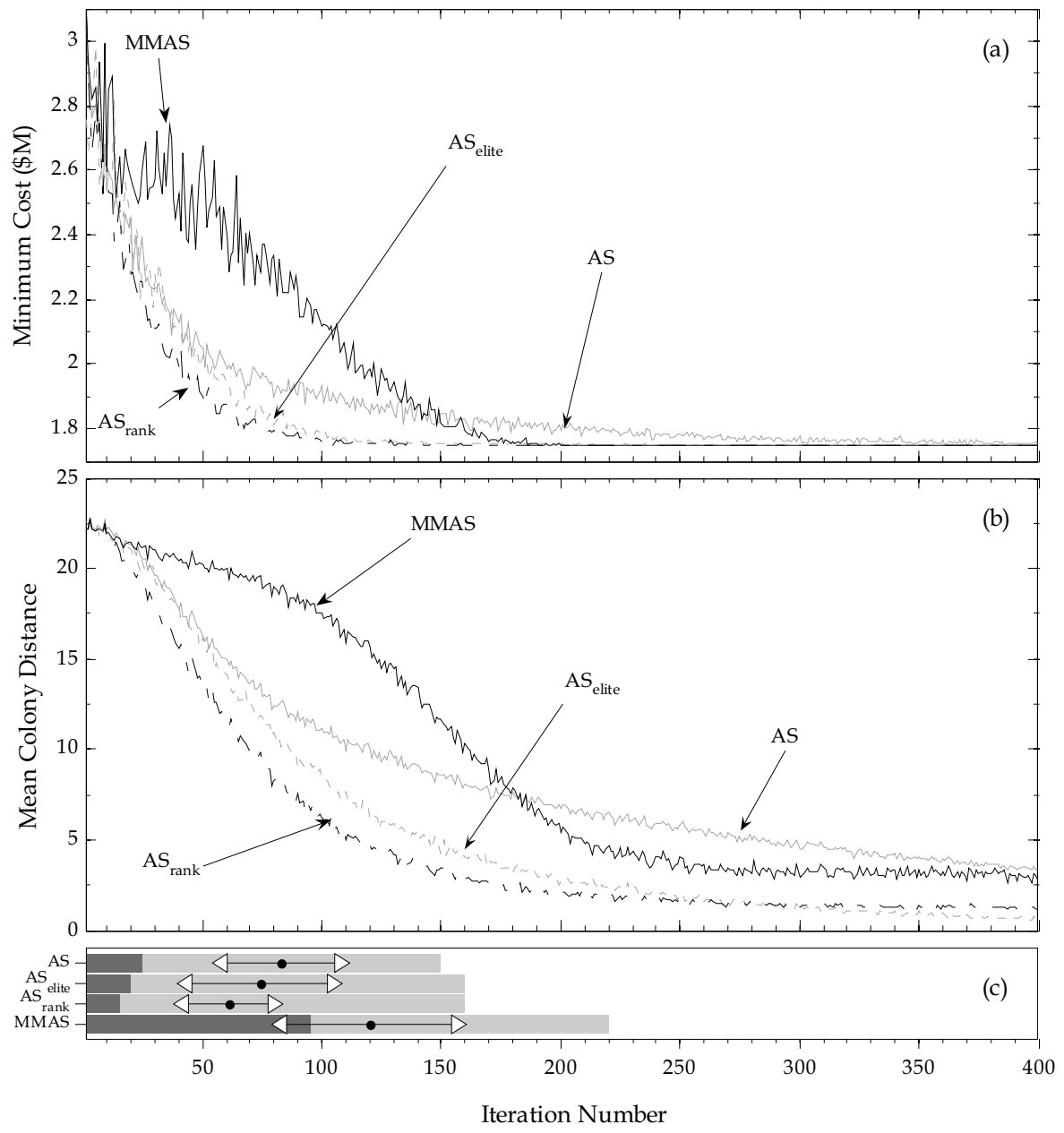

Figure 2. Plots of (a) the minimum cost (\$M) found in each iteration $f_{\min }(t)$, (b) the mean colony distance $d_{\Sigma}(t)$, and (c) run-time statistics for AS, AS elite, $\mathrm{AS}_{\text {rank, }}$ and MMAS applied to the Two Reservoir Problem. Plots (a) and (b) are averaged from 20 runs. Plot (c) depicts the three convergence phases: phase-I (dark grey); phase-II (light grey); phase-III (remaining white space). The line graphs overlaying the bar charts in (c) indicate the search-time statistics (based on 20 runs) with the dot indicating the mean search-time, and the left and right arrows indicating the mean minus and plus a standard deviation, respectively

The nature and time spent in each of these three phases is different for each algorithm. As seen in Figure 2, AS, $\mathrm{AS}_{\text {elite, }}$ and $\mathrm{AS}_{\text {rank }}$ have a relatively short broad searching phase-I, followed by a rapid convergence in phase-II. In contrast, MMAS has a relatively long broad 
searching phase-I, followed eventually by rapid phase-II convergence. The relatively long phase-I for MMAS may be attributed to the exploration encouraging mechanisms of pheromone bounding and pheromone smoothing. $\mathrm{AS}_{\text {elite }}$ and $\mathrm{AS}_{\text {rank }}$ have faster phase-II convergence than AS, which can possibly be attributed to the elitist exploitation mechanisms in these algorithms driving the search to converge faster. In phase-III, AS and $\mathrm{AS}_{\text {elite }}$ experience a gradually reducing, but steady, convergence, albeit $\mathrm{AS}_{\text {elite }}$ in a much tighter region after phase-II. In contrast to this, $\mathrm{AS}_{\text {rank }}$ and MMAS plateau in their convergence, as seen by $d_{\Sigma}(t)$ tending to a constant value in phase-III.

This difference in phase-III behaviour can be explained by a consideration of the pheromone adjustment operations of each algorithm. For ACO, convergence cannot only be defined in the context of the distribution of solutions throughout the solution space (i.e. the point at which $\left.d_{\Sigma}(t)=0\right)$, but also in a pheromone value context. That is, an ACO algorithm has converged when the pheromone value on all paths, except for a single path $S \in \mathcal{S}$, is effectively zero (i.e. zero for all computational purposes). At such a point, ants will only select edges from path $S$. For both $\mathrm{AS}$ and $\mathrm{AS}_{\text {elite, }}$ as pheromone values of paths become more dominant, the natural positive feedback process of the colony's path selection will dictate that the pheromone value on all edges, other than that of the increasingly dominant path, will decay to zero. Thus, these algorithms will converge to the point where $d_{\Sigma}(t)=0$. However, both $\mathrm{AS}_{\mathrm{rank}}$ and MMAS contain mechanisms that moderate this positive feedback process. Firstly, in the update process for $\mathrm{AS}_{\text {rank, }}$ in addition to the elitist ants, there are $\sigma-1$ unique paths that receive a weighted pheromone addition within each iteration. What this means for $\mathrm{AS}_{\text {rank }}$ is that there are always multiple paths for which the pheromone value does not decay to zero. Within MMAS, the pheromone bounding ensures that the pheromone values on all paths do not go below $\tau_{\min }(t)$.

The search-time statistics in Figure 2(c) (the triangle and dot lines plots superimposed over the bar charts) indicate the range of iteration numbers in which each algorithm found $S_{g b}$, the global best solution for the run. Interestingly, all four algorithms tended to find their global best solutions towards the end of phase-I and the beginning of phase-II, albeit MMAS at a later stage than the other three algorithms. AS elite $_{\text {and }} \mathrm{AS}$ had a greater variation in their search-times than $\mathrm{AS}_{\text {rank, }}$ with MMAS having the greatest variation in its search-times.

\subsection{Case Study 2: New York Tunnels Problem}

\subsubsection{Preliminaries}

The New York Tunnels Problem (NYTP) was first considered by Schaake and Lai (1969) while Dandy et al. (1996) was the first to apply an evolutionary algorithm to this problem. The network is a gravity system fed from a single reservoir, and consists of 20 nodes connected via 21 tunnels (Figure 3). There is a single demand case for the problem. Each tunnel has a null option, or the option to provide a duplicate tunnel with one of 15 different diameter sizes. The reader is referred to Dandy et al. (1996) for the case study details. This case study is the second smallest considered in this chapter, and has a search space of approximately $1.934 \times 10^{25}$ possible combinations.

\subsubsection{Results}

Based on the heuristics given in Table $1\left\{\tau_{0}, m\right\}=\{140,90\}$ and based on preliminary analyses $I_{\max }=500$ was found to be sufficient. A single run of the NYTP consisted of 45,000 function 
evaluations. The range of parameters tested was: $\sigma \in[2,80]$ for $\mathrm{AS}_{\text {elite; }} \sigma \in[2,80]$ for $\mathrm{AS}_{\text {rank }}$; $\left\{P_{\text {best }}, \delta\right\} \in\left[1 \times 10^{-5}, 0.99\right] \times[0,0.99]$ for MMAS. For $2 \leq \sigma \leq 20$ the performance of $\mathrm{AS}_{\text {elite }}$ varied less than $1 \%$, but for $\sigma>20$ the solution quality was increasingly worse. For AS rank, the performance varied less than $1 \%$ for the entire parameter range, with the better values being $8 \leq \sigma \leq 12$. For MMAS, the performance varied less than $1 \%$ for $0.005 \leq P_{\text {best }} \leq 0.99$ and $\delta \leq 0.0005$, with the solution quality degrading for lower values of $P_{b e s t}$ and higher values of $\delta$. The optimal parameter settings were as follows: $\sigma=8$ for $\mathrm{AS}_{\text {elite; }} \sigma=8$ for $\mathrm{AS}_{\text {rank; }}$; $\left\{P_{\text {best }}, \delta\right\}=\left\{0.05,5 \times 10^{-5}\right\}$ for MMAS.

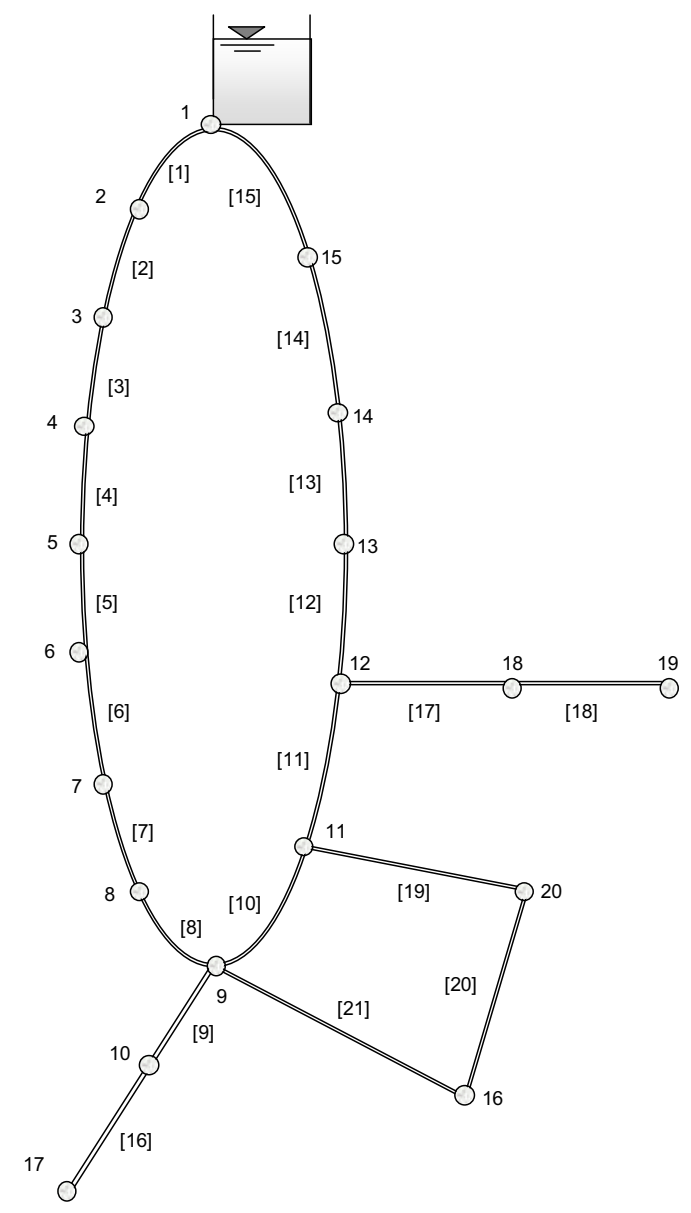

Figure 3. Network layout for New York Tunnels Problem

Table 3 gives a comparison of the performance of the ACO algorithms considered in this paper with that of the current best performing algorithms from the literature for the NYTP. A detailed statistical analysis of these algorithms was given in Zecchin et al. (2007), but all algorithms performed well, with $\mathrm{AS}_{\text {elite, }} \mathrm{AS}_{\text {rank }}$ and MMAS, on average, finding solutions within a $1 \%$ cost of the known-optimum. 
Case Study Based Convergence Behaviour Analysis of ACO Applied

\begin{tabular}{|c|c|c|c|c|c|c|c|}
\hline \multirow{3}{*}{$\begin{array}{l}\text { Algorithm } \\
\text { AS }\end{array}$} & \multicolumn{6}{|c|}{ Best-cost (\$M) (\% deviation from optimum) } & \multirow{3}{*}{$\begin{array}{c}\text { Mean search-time } \\
\text { (evaluation no.) }\end{array}$} \\
\hline & \multicolumn{2}{|c|}{ Minimum } & \multicolumn{2}{|c|}{ Mean } & \multicolumn{2}{|c|}{ Maximum } & \\
\hline & 39.204 & $(1.47)$ & 39.910 & $(3.29)$ & 40.922 & $(5.91)$ & \\
\hline $\mathrm{AS}_{\text {elite }}$ & 38.638 & $(0.00)$ & 38.988 & $(0.91)$ & 39.511 & $(2.26)$ & 21,945 \\
\hline $\mathrm{AS}_{\text {rank }}$ & 38.638 & $(0.00)$ & 38.777 & $(0.36)$ & 39.221 & $(1.51)$ & 19,319 \\
\hline MMAS & 38.638 & $(0.00)$ & 38.836 & $(0.51)$ & 39.415 & $(2.01)$ & 30,711 \\
\hline PEa & 41.800 & $(8.18)$ & \multicolumn{2}{|c|}{-} & \multicolumn{2}{|c|}{-} & NA \\
\hline $\mathrm{GA}_{\mathrm{imp}} \mathrm{b}$ & 38.796 & $(0.41)$ & \multicolumn{2}{|c|}{ NA } & \multicolumn{2}{|c|}{ NA } & 96,750 \\
\hline $\mathrm{GA}^{\mathrm{c}}$ & $37.13^{\mathrm{i}}$ & - & \multicolumn{2}{|c|}{ NA } & \multicolumn{2}{|c|}{ NA } & $\sim 1,000,000$ \\
\hline $\operatorname{ACOA}_{\text {i-best }}{ }^{\mathrm{d}}$ & 38.638 & $(0.00)$ & \multicolumn{2}{|c|}{ NA } & \multicolumn{2}{|c|}{ NA } & 13,928 \\
\hline TSe & $37.13^{\mathrm{i}}$ & - & \multicolumn{2}{|c|}{ NA } & \multicolumn{2}{|c|}{ NA } & $\sim 10,000$ \\
\hline$A S_{\text {i-best }}{ }^{\mathrm{f}}$ & 38.638 & $(0.00)$ & 38.849 & $(0.55)$ & 39.492 & $(2.21)$ & 22,052 \\
\hline ACSg & 38.638 & $(0.00)$ & 39.629 & (2.57) & 41.992 & $(8.68)$ & 23,972 \\
\hline $\mathrm{GA}_{\text {adapt }}{ }^{\mathrm{h}}$ & 38.638 & $(0.00)$ & 38.770 & $(0.34)$ & 39.07 & (1.12) & 15,680 \\
\hline \multicolumn{8}{|c|}{$\begin{array}{l}\text { a Partial enumeration (Gessler, 1982). b Improved GA that used a variable exponent in fitness scaling, an } \\
\text { adjacency mutation operator, and Gray code representation (Dandy et al. 1996). c Genetic algorithm } \\
\text { (Savic \& Walters, 1997). d Iteration-best updating version of ACO (Maier, et al. 2002). e Tabu search } \\
\text { (Cunha \& Ribeiro, 2004). }{ }^{\mathrm{f}} \text { An improved iteration-best version of AS (Zecchin et al. 2005). } \text { g }^{\mathrm{g}} \text { Ant colony } \\
\text { system (Zecchin et al. 2007). }{ }^{\text {h }} \text { Parameter free, self-adapting, boundary searching genetic algorithm } \\
\text { (Afshar \& Marino, 2007). }{ }^{\mathrm{i}} \text { Not assessed as feasible by EPANET2 (Maier et al., 2002). }\end{array}$} \\
\hline
\end{tabular}

Table 3. Comparison of performance of AS, ACS, AS elite, AS rank, MMAS, and other algorithms from the literature applied to the New York Tunnels Problem. Results for AS, $\mathrm{AS}_{\text {elite, }} \mathrm{AS}_{\text {rank, }}$ and MMAS are based on 20 runs. NA means that the information was not available

Plots of the iteration best-costs $f_{\text {min }}(t)$, the mean-colony-distance $d_{\Sigma}(t)$, and the searching phases and search-time statistics for the algorithms applied to the NYTP are given in Figure 4(a)-(c). Again, the three distinct searching phases observed for the TRP are observed in the behaviour of $f_{\min }$ and $d_{\Sigma}$. The relative behaviours of the algorithms applied to the NYTP are similar to that for the TRP, except for the faster convergence of AS in phase-I than that of both $\mathrm{AS}_{\text {elite }}$ and $\mathrm{AS}_{\text {rank. }}$. The effectiveness of the additional pheromone adjustment mechanisms in $\mathrm{AS}_{\text {elite, }} \mathrm{AS}_{\text {rank }}$ and MMAS is made clear in Figure 4(a). This is seen by the fact that, for the majority of the phase-III searching, these algorithms have confined the search to 
the high quality region of the solution space, as indicated by the near optimal $f_{\min }$. In contrast, by the end of the run-time, AS has converged to a smaller region than MMAS, but the higher value of $f_{\min }$ indicates that the search is not in a near optimal region. This behaviour is also exhibited in the performance statistics of Table 3 .

Figure 4(c) gives a plot of the search-time statistics (the distribution of iteration times taken by the algorithms to find their global best run-time cost $S_{g b}$ ) for the NYTP. In contrast to the TRP, the algorithms tended to find their $S_{g b}$ relatively later in the searching phases. $A S_{\text {elite }}$ and $\mathrm{AS}_{\text {rank }}$ tended to find their best cost just after the descent in phase-II, whereas AS and MMAS found their best cost well into phase-III. This observation is interesting, as even though $\mathrm{AS}_{\text {elite }}$ and $\mathrm{AS}_{\text {rank }}$ had clearly not converged (as $d_{\Sigma}>0$ ), and their search was intensified within a near optimal region of the solution space, they were both unable to find higher quality solutions in phase-III of their search. The implications of this are that the phase-III search for $\mathrm{AS}_{\text {elite }}$ and $\mathrm{AS}_{\text {rank }}$ is not an effective phase in their searching behaviour.

\subsection{Case Study 3: Hanoi Problem}

\subsubsection{Preliminaries}

The Hanoi Problem (HP), first published by Fujiwara and Khang (1990), has been considered by numerous authors in its discrete problem formulation (Savic \& Walters 1997; Cunha \& Sousa 1999; Wu et al. 2001). This case study is for a new design that consists of 34 pipes and 32 nodes organised in three loops (Figure 5). The system is gravity fed by a single reservoir and has only a single demand case. For each link, there are six different new pipe options where a minimum diameter constraint is enforced. For case study details, the reader is referred to Cunha \& Sousa (1999). This case study is the second largest considered in this chapter, having a problem size of approximately $2.87 \times 10^{26}$ combinations.

Based on the heuristics given in Table $1\left\{\tau_{0}, m\right\}=\{26,80\}$ and $I_{\max }=1,500$ were found to be sufficient, implying that a single run for the HP consisted of 120,000 function evaluations. The range of parameters tested was: $\sigma \in[2,70]$ for $\mathrm{AS}_{\text {elite }} \sigma \in[2,70]$ for $\mathrm{AS}_{\text {rank }}$; for MMAS, $\left\{P_{\text {best }}, \delta\right\} \in\left[1 \times 10^{-5}, 0.99\right] \times[0,0.005]$. In general, the performances of $\mathrm{AS}_{\text {elite, }} \mathrm{AS}_{\text {rank, }}$ and MMAS were much more sensitive to their respective parameter settings for this case study, such that only moderate variations from the parameters selected resulted in the inability to find feasible solutions for some runs. For $\mathrm{AS}_{\text {elite, }}$ no feasible solutions were found for $\sigma \leq 10$, with the best performance occurring with $\sigma=40$. For values of $\sigma>20$, no feasible solutions

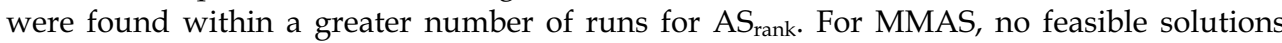
were found for $P_{\text {best }} \leq 0.1$ and $\delta \geq 0.001$, however, there was a less than $1 \%$ variation in solution quality for $0.5 \leq P_{\text {best }} \leq 0.65$. The selected parameter values were as follows: for $\mathrm{AS}_{\text {elite, }} \sigma=40$; for $\mathrm{AS}_{\text {rank, }} \sigma=20$; for MMAS, $\left\{P_{\text {best }}, \delta\right\}=\{0.5,0\}$. An important point to note is that the best parameter settings for this case study vary greatly from those of all the other case studies. A common thread is that the optimal parameter settings for this case study increased each of the algorithms' emphasis on exploitation. For example: for $\mathrm{AS}_{\text {elite, }}$ and to a lesser degree $\mathrm{AS}_{\mathrm{rank}}$, the number of elitist ants for this case study was far greater than for the other case studies; for MMAS, $P_{\text {best }}$ was higher (indicating looser pheromone bounds) and $\delta$ was set to a low value, both of these indicating a reduction in exploration potential. Despite this notable sensitivity, the parameter heuristics proposed by Zecchin et al. (2005) resulted in extremely good performance for MMAS and $\mathrm{AS}_{\text {rank. }}$ 


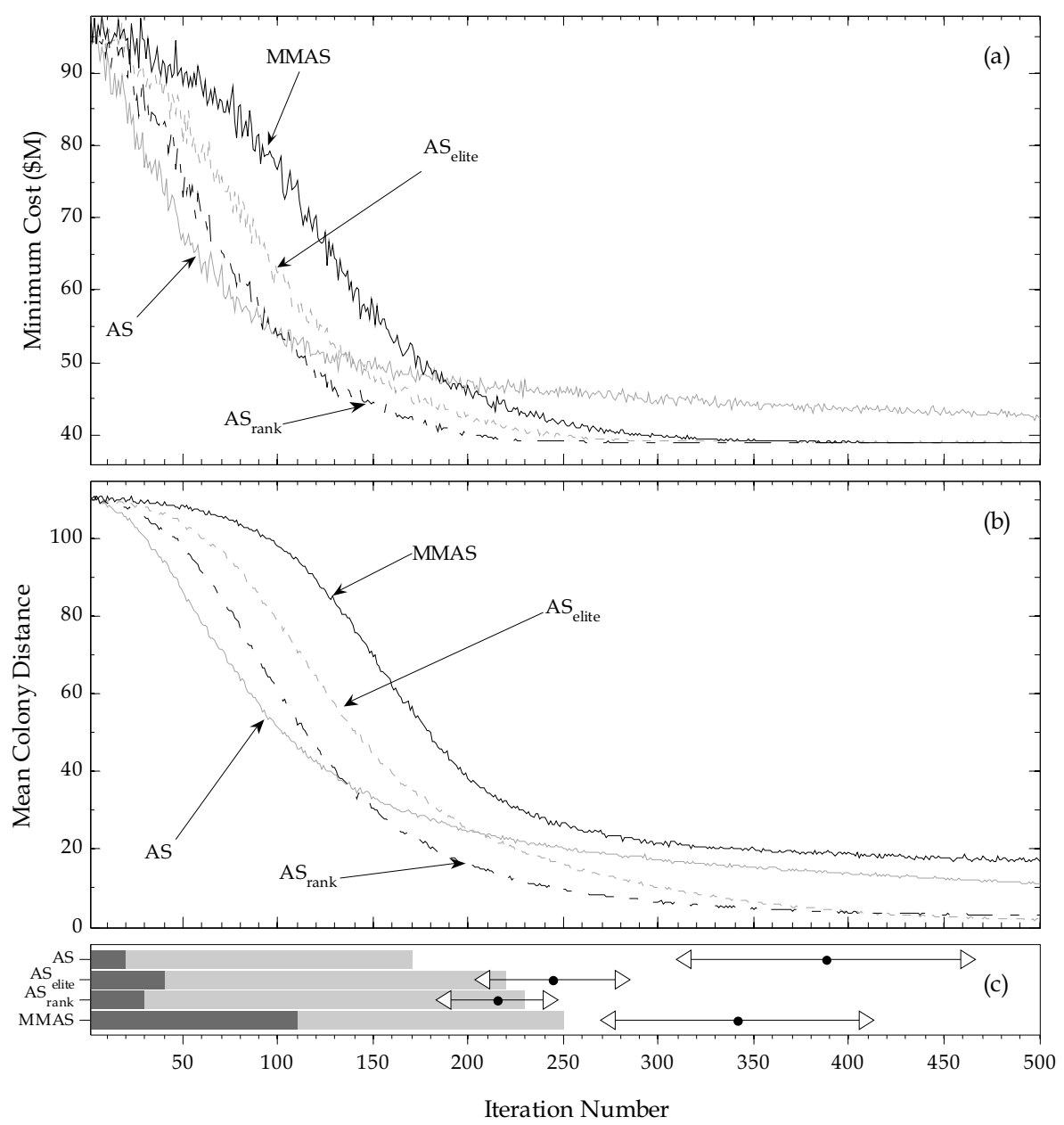

Figure 4. Plots of (a) the minimum cost $(\$ \mathrm{M})$ found in each iteration $f_{\min }(t)$, (b) the mean

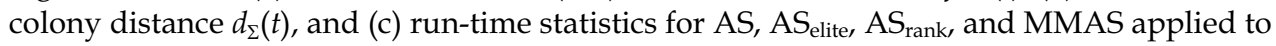
the New York Tunnels Problem. Plots (a) and (b) are averaged from 20 runs. Plot (c) depicts the three convergence phases: phase-I (dark grey); phase-II (light grey); phase-III (remaining white space). The line graphs overlaying the bar charts in (c) indicate the search-time statistics (based on 20 runs) with the dot indicating the mean search-time, and the left and right arrows indicating the mean minus and plus a standard deviation, respectively 


\subsubsection{Results}

Table 4 shows a comparison of the performance of the ACO algorithms with the other best performing algorithms in the literature. A detailed statistical analysis of the algorithms was given in Zecchin et al. (2007), but as a summary, MMAS, and to a lesser extent $\mathrm{AS}_{\text {rank, were }}$ the only algorithms that performed well on the HP. AS elite was unable to find high quality solutions, and AS was not able to find any feasible solutions. Other authors have also noted that the HP has a small feasible region (Eusuff \& Lansey 2003).

13<smiles>O=CO</smiles>

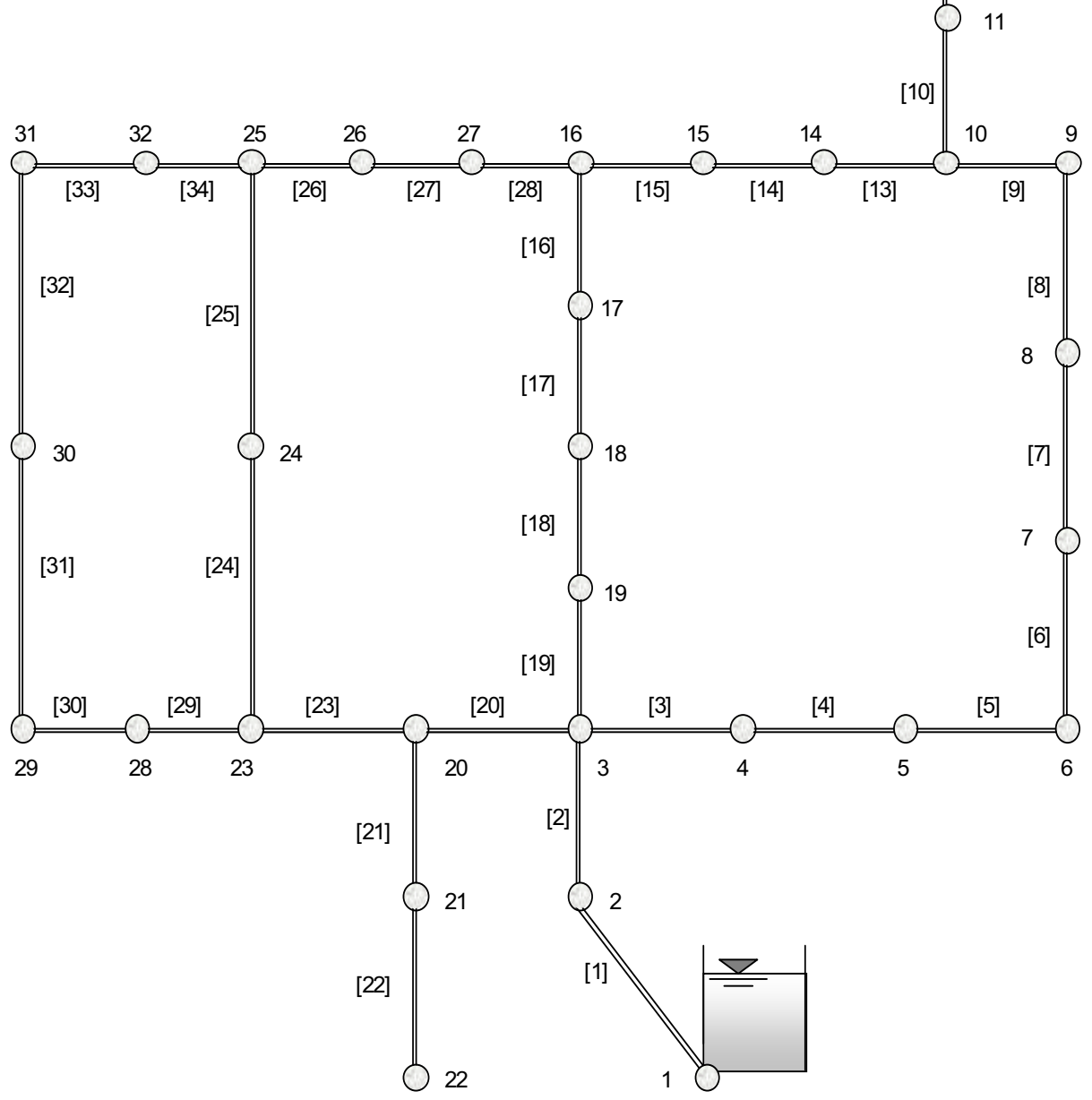

Figure 5. Network layout for the Hanoi Problem 


\begin{tabular}{|c|c|c|c|c|c|c|c|}
\hline \multirow{2}{*}{ Algorithm } & \multicolumn{6}{|c|}{ Best-cost (\$M) (\% deviation from optimum) } & \multirow{2}{*}{$\begin{array}{l}\text { Mean search-time } \\
\text { (evaluation no.) }\end{array}$} \\
\hline & \multicolumn{2}{|c|}{ Minimum } & \multicolumn{2}{|c|}{ Mean } & \multicolumn{2}{|c|}{ Maximum } & \\
\hline AS & \multicolumn{2}{|c|}{ NFS } & \multicolumn{2}{|c|}{ NFS } & \multicolumn{2}{|c|}{ NFS } & - \\
\hline $\mathrm{AS}_{\text {elite }}$ & 6.827 & $(11.30)$ & 7.295 & (18.93) & 8.187 & $(33.48)$ & 59,917 \\
\hline $\mathrm{AS}_{\text {rank }}$ & 6.206 & $(1.17)$ & 6.506 & $(6.07)$ & 6.788 & $(10.66)$ & 75,328 \\
\hline MMAS & 6.134 & $(0.00)$ & 6.394 & $(4.24)$ & 6.635 & $(8.17)$ & 85,571 \\
\hline $\mathrm{GA}^{\mathrm{a}}$ & 6.195 & $(1.00)$ & \multicolumn{2}{|c|}{ NA } & \multicolumn{2}{|c|}{ NA } & $\sim 1,000,000$ \\
\hline $\mathrm{SA}^{\mathrm{b}}$ & $6.053 \mathrm{~g}$ & - & \multicolumn{2}{|c|}{ NA } & \multicolumn{2}{|c|}{ NA } & $\sim 53,000$ \\
\hline $\mathrm{GA}_{\mathrm{fm}}{ }^{\mathrm{c}}$ & 6.182 & $(0.78)$ & \multicolumn{2}{|c|}{ NA } & \multicolumn{2}{|c|}{ NA } & 113,626 \\
\hline TSd & $6.053 \mathrm{~g}$ & - & \multicolumn{2}{|c|}{ NA } & \multicolumn{2}{|c|}{ NA } & $\sim 10,000$ \\
\hline$A S_{\text {i-best }}{ }^{\mathrm{e}}$ & 6.367 & (3.80) & 6.842 & (11.54) & 7.474 & (21.95) & 67,136 \\
\hline $\mathrm{ACS}^{\mathrm{f}}$ & 7.754 & (26.41) & 8.109 & (32.20) & 8.462 & (37.96) & 61,324 \\
\hline \multicolumn{8}{|c|}{$\begin{array}{l}\text { a Genetic algorithm (Savic \& Walters, 1997). b Simulated annealing (Cunha \& Sousa, 1999). c The fast } \\
\text { messy genetic algorithm (Wu et al., 2001). d Tabu search (Cunha \& Ribeiro, 2004). e An iteration-best } \\
\text { pheromone updating version of AS (Zecchin et al. 2005); f Ant colony system (Zecchin et al. 2007). } \\
\text { g Infeasible by EPANET2 (Zecchin et al., 2005). }\end{array}$} \\
\hline
\end{tabular}

Table 4. Comparison of performance of AS, ACS, $\mathrm{AS}_{\text {elite, }} \mathrm{AS}_{\text {rank, }}$ MMAS, and other algorithms from the literature applied to the Hanoi Problem. Results for AS, $\mathrm{AS}_{\text {elite, }} \mathrm{AS}_{\text {rank, }}$ and MMAS are based on 20 runs. NFS means no feasible solution was found, NA means that the information was not available

Plots of the iteration best-costs $f_{\min }(t)$, the mean-colony-distance $d_{\Sigma}(t)$, and the searching phases and search-time statistics for the algorithms applied to the HP are given in Figure 6(a)-(c). Vastly different behaviours were observed for the HP in comparison to the other case studies, but the three phases of searching are still distinct. A marked difference though is the relative lengths of the phase-I searching, (AS, $\mathrm{AS}_{\text {elite, }}$ and $\mathrm{AS}_{\text {rank }}$ all have a far longer phase-I search than MMAS) and the distinct hump in the phase-II convergence of MMAS. The differences in the trends of $f_{\min }$ and $d_{\Sigma}$ for this case study can be explained by the small feasible region. The maximum cost of a feasible solution for the HP, which is also the maximum network cost (for which the penalty cost $P C$ from (15) is zero), is $C\left(\Omega^{\max }\right)=\$ \mathrm{M}$ 10.96. Therefore, any solutions with costs higher than this are infeasible. With this in mind, the appearance of the data points of $f_{\min }$ in Figure 6(a) can be interpreted as the iteration times at which each algorithm first found feasible solutions. This is why no plot of $f_{\min }$ for AS is seen in Figure 6(a), as AS found no feasible solutions for the HP. 


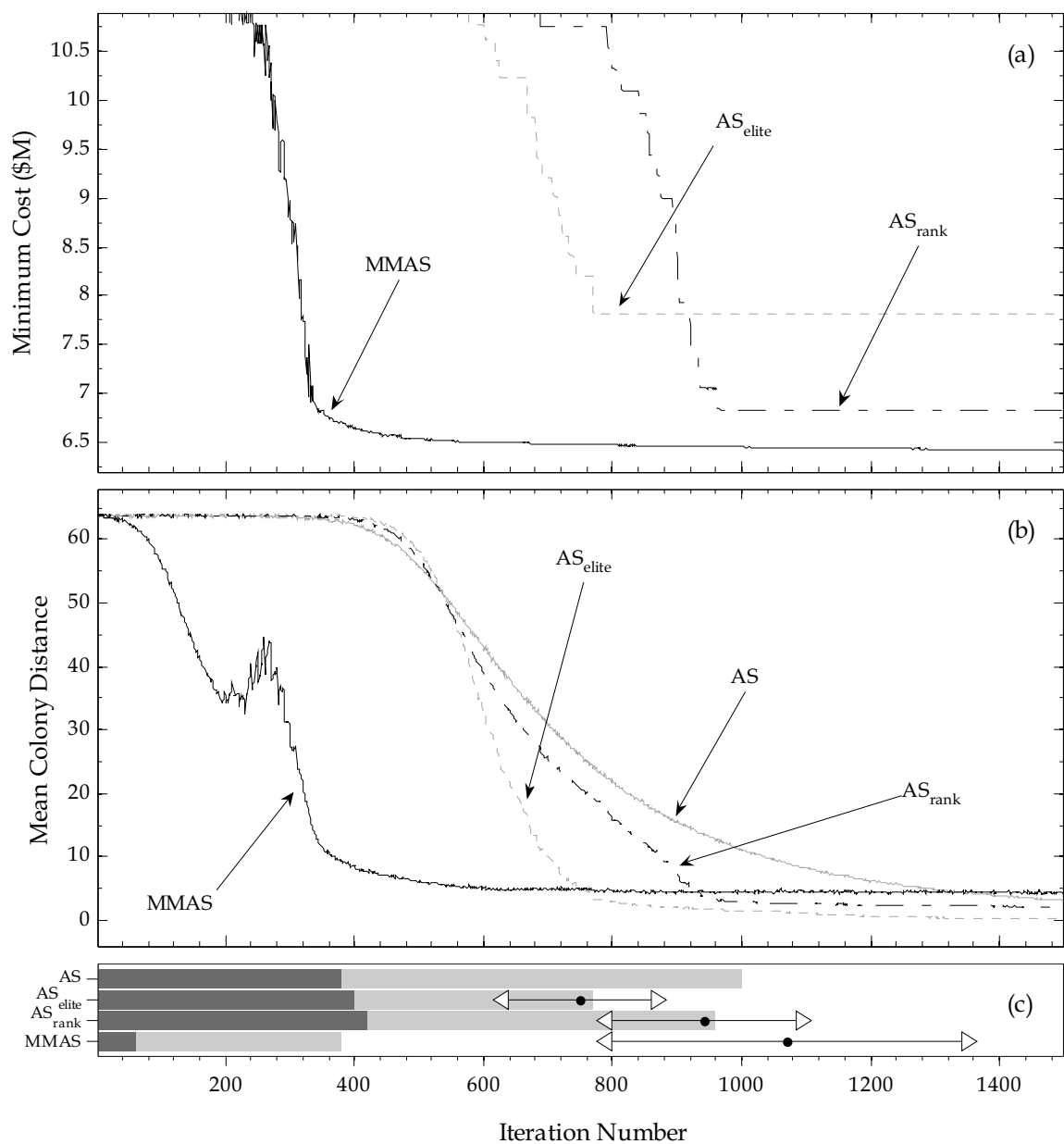

Figure 6. Plots of (a) the minimum cost $(\$ M)$ found in each iteration $f_{\min }(t)$, (b) the mean colony distance $d_{\Sigma}(t)$, and (c) run-time statistics for AS, AS elite, $\mathrm{AS}_{\text {rank, }}$ and MMAS applied to the Hanoi Problem. Plots (a) and (b) are averaged from 20 runs. Plot (c) depicts the three convergence phases: phase-I (dark grey); phase-II (light grey); phase-III (remaining white space). The line graphs overlaying the bar charts in (c) indicate the search-time statistics (based on 20 runs) with the dot indicating the mean search-time, and the left and right arrows indicating the mean minus and plus a standard deviation, respectively

This entry into the feasible region for $\mathrm{AS}_{\text {elite, }} \mathrm{AS}_{\text {rank }}$ and MMAS occurred well into the phase-II search (for MMAS, it is seen to coincide with the hump in the phase-II convergence). This means that the phase-I search was entirely within the infeasible region, and furthermore, it took the algorithms some time to effectively use the information in the infeasible region before they could begin the phase-II convergence to guide the search into the feasible region. Interestingly, only the algorithms that contain exploitive mechanisms were able to find the feasible region. 
Considering the search-time statistics in Figure 6(c), it is seen that $\mathrm{AS}_{\text {elite }}$ and $\mathrm{AS}_{\text {rank }}$ found their best-costs towards the end of the phase-II search, with $\mathrm{AS}_{\text {rank }}$ consistently finding higher quality solutions than $\mathrm{AS}_{\text {elite. }}$. For MMAS however, the best-cost was found well into the phaseIII search, again illustrating the effectiveness of MMAS's confined phase-III searching.

\begin{tabular}{|c|c|c|c|c|c|c|c|}
\hline \multirow{3}{*}{$\begin{array}{c}\text { Algorithm } \\
\text { AS }\end{array}$} & \multicolumn{6}{|c|}{ Best-cost (\$M) (\% deviation from optimum) } & \multirow{3}{*}{\begin{tabular}{|c}
$\begin{array}{c}\text { Mean search-time } \\
\text { (evaluation no.) }\end{array}$ \\
131,769
\end{tabular}} \\
\hline & \multicolumn{2}{|c|}{ Minimum } & \multicolumn{2}{|c|}{ Mean } & \multicolumn{2}{|c|}{ Maximum } & \\
\hline & 80.855 & $(4.63)$ & 83.572 & $(8.15)$ & 85.267 & (10.34) & \\
\hline $\mathrm{AS}_{\text {elite }}$ & 77.922 & $(0.84)$ & 79.806 & $(3.28)$ & 81.986 & $(6.10)$ & 90,404 \\
\hline $\mathrm{AS}_{\text {rank }}$ & 77.434 & $(0.21)$ & 78.492 & $(1.58)$ & 79.863 & $(3.35)$ & 72,276 \\
\hline MMAS & 77.275 & $(0.00)$ & 78.213 & $(1.21)$ & 79.353 & (2.69) & 238,264 \\
\hline$A S_{\text {i-best }}{ }^{\mathrm{a}}$ & 77.275 & $(0.00)$ & 78.302 & (1.33) & 79.922 & (3.43) & 75,760 \\
\hline $\mathrm{ACSb}$ & 77.275 & $(0.00)$ & 80.586 & $(4.28)$ & 86.682 & (12.17) & 471,977 \\
\hline
\end{tabular}

Table 5. Comparison of performance of AS, $\mathrm{AS}_{\text {elite, }} \mathrm{AS}_{\mathrm{rank}}, \mathrm{MMAS}$, and other algorithms from the literature applied to the Doubled New York Tunnels Problem. Results for AS, $\mathrm{AS}_{\text {elite, }} \mathrm{AS}_{\text {rank, }}$ and MMAS are based on 20 runs

\subsection{Case Study 4: Doubled New York Tunnels Problem}

\subsubsection{Preliminaries}

The Doubled New York Tunnels Problem (2-NYTP), first studied in Zecchin et al. (2005), consists of two NYTP networks connected via the single reservoir at node 1 in Figure 3 . The link and node details are as for the NYTP. This problem has a search space size of $3.741 \mathrm{x}$ $10^{50}$ and is the largest case study considered in this chapter.

\subsubsection{Results}

Based on the heuristics given in Table $1,\left\{\tau_{0}, m\right\}=\{200,170\}$, and from a preliminary analysis, $I_{\max }=3,000$, therefore, a single run for the 2-NYTP consisted of 510,000 function evaluations. The range of parameters tested was: $\sigma \in[1,160]$ for $\mathrm{AS}_{\text {elite; }} \sigma \in[2,160]$ for $\mathrm{AS}_{\text {rank; }}$ for MMAS, $\left\{P_{\text {best }}, \delta\right\} \in\left[1 \times 10^{-6}, 0.99\right] \times[0,0.99]$.

$\mathrm{AS}_{\text {elite }}$ achieved a less than $1 \%$ variation in the performance for $1 \leq \sigma \leq 5$, with the solution quality deteriorating for higher values of $\sigma$. AS rank's performance varied less than $5 \%$ for the entire parameter range of $\sigma$, with the best values occurring for $6 \leq \sigma \leq 10$. For MMAS, the performance varied less than $1 \%$ for $0.0005 \leq P_{\text {best }} \leq 0.5$ and $\delta \leq 0.0001$, with the solution quality degrading for parameter values outside these ranges. The selected parameter values were: $\sigma=3$ for $\mathrm{AS}_{\text {elite }} \sigma=8$ for $\mathrm{AS}_{\text {rank; }}\left\{P_{\text {best }}, \delta\right\}=\{0.001,0\}$ for MMAS.

Table 5 shows a comparison of the performance of the ACO algorithms considered for the 2-NYTP with that obtained using other algorithms from the literature. A detailed statistical analysis of these algorithms was given in Zecchin et al. (2007), but as a summary, AS $_{\text {rank }}$ and MMAS performed consistently well (the former with extremely computationally efficient 
search times), with $\mathrm{AS}_{\text {elite }}$ performing only moderately inferior to that the above two, algorithms but significantly better than AS.
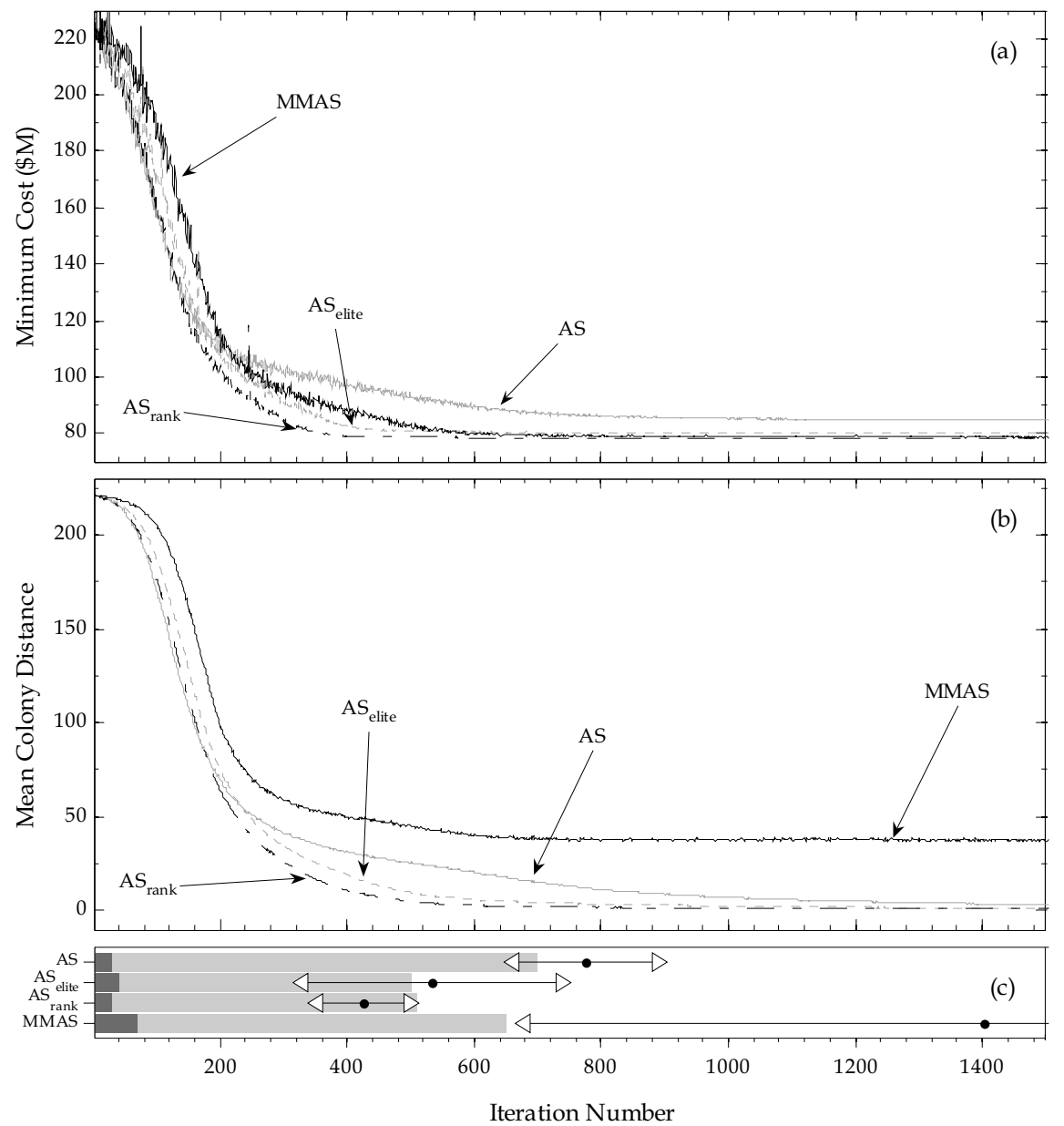

Figure 7. Plots of (a) the minimum cost $(\$ \mathrm{M})$ found in each iteration $f_{\min }(t)$, (b) the mean colony distance $d_{\Sigma}(t)$, and (c) run-time statistics for AS, AS elite, $\mathrm{AS}_{\text {rank, }}$ and MMAS applied to the Doubled New York Tunnels Problem. Plots (a) and (b) are averaged from 20 runs. Plot (c) depicts the three convergence phases: phase-I (dark grey); phase-II (light grey); phase-III (remaining white space). The line graphs overlaying the bar charts in (c) indicate the searchtime statistics (based on 20 runs) with the dot indicating the mean search-time, and the left and right arrows indicating the mean minus and plus a standard deviation, respectively

Plots of the iteration best-costs $f_{\text {min }}(t)$, the mean-colony-distance $d_{\Sigma}(t)$, and the searching phases and search-time statistics for the algorithms applied to the 2-NYTP are given in Figure 7(a)-(c). These figures show behaviour quite similar to that for the NYTP, just on a larger time scale. The main difference between the two is the relative size of $d_{\Sigma}$ in MMAS's phase-III search with 
respect to the other algorithms. In other words, MMAS still seems to be searching a relatively broad area of the solution space in phase-III. This explorative behaviour is seen as being effective, as the distribution of search-times in Figure 7(c) shows that, as with the other case studies, MMAS finds many of its best-cost solutions in this phase of the search.

\subsection{Discussion}

As in a previous study (Zecchin et al. 2007), the additional pheromone adjustment mechanisms in $\mathrm{AS}_{\text {elite, }} \mathrm{AS}_{\text {rank }}$ and MMAS were shown to improve the performance of ACO, in comparison to AS, when applied to the WDSP. $\mathrm{AS}_{\text {elite }}$ and $\mathrm{AS}_{\text {rank }}$ were seen to be relatively fast algorithms, finding high quality solutions for all case studies except the difficult HP. MMAS was observed as a slower algorithm, but able to consistently find high quality solutions for all the case studies considered. Below is a discussion of the convergence behaviour of each algorithm as observed in the case studies considered.

AS was observed to converge quickly initially (i.e. typically a short phase-I search), with an accompanied increase in solution quality. However, by the end of its (also relatively short) phase-II, AS's search was still typically broad, and it did not seem to be able to focus the colony to find solutions that were as good as those found by the other algorithms. AS was also not able to find the feasible region at all for the HP.

Despite $\mathrm{AS}_{\text {elite }} \mathrm{S}$ emphasis on exploitation, its phase-II convergence was not consistently faster than that of AS, implying an initially more explorative phase-I. The exploitative nature of $\mathrm{AS}_{\text {elite }}$ was seen at the end of phase-II, at which point $\mathrm{AS}_{\text {elite }}$ converged to a comparatively small region of high quality solutions. AS elite tended to find its best-cost solutions towards the end of phase-II and at the beginning of phase-III. AS elite's ability to find the feasible region for the HP can be directly attributed to the exploitive nature of its elitist ants, which effectively used the information in the infeasible region to guide the search into the feasible region. However, once in the feasible region, $\mathrm{AS}_{\text {elite }}$ was not able to find high quality solutions.

$\mathrm{AS}_{\text {rank }}$ consistently converged faster than $\mathrm{AS}_{\text {elite, }}$ and to a tighter searching region (lower $d_{\Sigma}$ ), by the end of phase-II. AS rank had a typically lower, and less variable, search-time than the other algorithms, with best-cost solutions generally found towards the end of phase-II. Given its speed of convergence, and the high quality of the solutions found, $\mathrm{AS}_{\text {rank }}$ 's pheromone updating scheme, although highly exploitative, is considered to be very effective. However, in situations, such as the small feasible region of the HP or the relatively large solution space of the 2-NYTP, AS $_{\text {rank }}$ 's inability to conduct an explorative phase-III search, means that it does not perform as well as MMAS for these case studies.

MMAS typically had the longest phase-I search, which can be attributed to its exploration encouraging pheromone bounding and pheromone smoothing mechanisms. MMAS maintained a relatively broad search after its phase-II convergence, but unlike AS, it was still able to find high quality solutions. This aspect of relatively broad phase-III searching, coupled with high solution quality, can be attributed to MMAS's effective management of exploitation, by updating only the iteration best path, and exploration, by lower bounding all paths' pheromone values. As such, MMAS tended to find its best cost solutions well into phase-III. This lower bounding of the pheromone values also explains MMAS's ability to effectively explore the feasible region of the HP. As the discovery of the higher quality solutions in the feasible region raised the lower pheromone bound once MMAS entered the feasible region, the pheromone values were all partially replenished, with the information concerning the feasible region being retained. From this perspective, the hump observed in Figure 6(b) can 
be considered as another phase-I period for the MMAS search as it entered the feasible region. That is, due to the replenishing nature of the lower pheromone bound, the searching phases seemed to start again once the feasible region was found.

\section{Conclusions}

To gain a more complete understanding of ACO algorithms, it is important to not only consider their performance with respect to their solution quality and computational efficiency, but also the algorithms' searching behaviour. In this chapter, two statistics of searching behaviour have been considered, (i) the minimum cost found within an iteration, which is an indication of search quality, and (ii) the mean colony distance, a topological measure that describes the spread of solutions through the solution space and thus provides an indication of the degree of convergence of an algorithm.

Four ACO algorithms were considered in this chapter, namely, Ant System (AS), Elitist Ant System (AS elite $)$ Elitist-Rank Ant System $\left(\mathrm{AS}_{\text {rank }}\right)$, and Max-Min Ant System (MMAS). The focus of this chapter was a case study based computational analysis of the convergence behaviour of these four algorithms. The problem type considered was the water distribution system problem, a classical combinatorial optimisation problem in the field of civil engineering. The case studies considered were the Two-Reservoir Problem (TRP), the New York Tunnels Problem (NYTP), the Hanoi Problem (HP), and the Doubled New York Tunnels Problem (2-NYTP).

From studying the convergence behaviour as exhibited by the mean colony distance, three distinct searching phases were observed for all algorithms. Phase-I was observed to be a broad searching phase in which only low quality solutions were found. In phase-II, a rapid convergence was observed, where increasingly good solutions were found and the colonies search was guided into smaller and higher quality regions of the solution space. Phase-III consisted of compact and dense searching in the high quality regions discovered in phase-II, where the convergence rate was much reduced.

Each algorithm exhibited different behaviour in each phase. These differences were interpreted from the perspective of the algorithms' formulations. For example, the exploitative algorithms, $\mathrm{AS}_{\text {elite }}$ and $\mathrm{AS}_{\text {rank }}$ experienced an extremely short phase-I, followed by a rapid convergence in phase-II. In contrast, the exploration encouraging MMAS had a significantly longer phase-I search and tended to converge to a much broader region than the other algorithms at the end of phase-II. AS tended to converge quickly initially, but its lack of exploitative mechanisms meant that, even by the end of phase-II, it was not able to focus its search in the high quality regions of the search space.

Combining this qualitative three phase description with the search-time statistics (the time in the search at which the algorithms found their best-cost) leads to a deeper understanding of the productive stages in the algorithms' search. In almost all instances, AS $_{\text {rank' }}$ 's search-time occurred near the end of phase-II. What this means is that the phase-III searching for $\mathrm{AS}_{\text {rank }}$ was seen to be unproductive. $\mathrm{AS}_{\text {elite }}$ performed similarly to $A \mathrm{~S}_{\text {rank, }}$ but with slightly longer search-times. The search-times for the explorative MMAS were typically much longer than those of the others algorithms, and the best-cost solutions tended to occur in the phase-III searching stage. The implications of this are that, even though MMAS maintained a relatively broad phase-III search, this longer term exploration was fruitful as solutions of higher quality were found. 
This chapter illustrates how relatively simple run-time statistics can yield significant insight into the convergence behaviour of ACO algorithms. The type of analysis presented in this chapter shows potential to be useful for the purpose of both research and application. In terms of research (and algorithmic development), considering run-time behavioural statistics could aid in understanding the behavioural impacts of algorithmic mechanisms, and also provide a more informative comparison of different algorithms. In terms of application, run-time statistics could aid in understanding the influence of parameter variations, and facilitate the determination of appropriate parameter values, both online and offline.

\section{References}

Afshar, M.H., \& Marino, M.A. (2007). A parameter-free self-adapting boundary genetic search for pipe network optimization. Comput. Optim. App., 37, 83-102

Boese, K. D.; A. B. Kahng, \& S.Muddu (1994). A new adaptive multi-start technique for combinatorial global optimization. Operations Research Letters, 16, 2, 101-113

Bullnheimer, B.; Hartl, R. F. \& Strauss, C. (1999). A new rank based version of the Ant System: A computational study. Central European Journal for Operations Research and Economics, 7, 1, 25-38

Coello Coello, C.A. (2002). Theoretical and numerical constraint-handling techniques used with evolutionary algorithms: A survey of the state of the art, Computer methods in applied mechanics and engineering, 191, 1245-1287

Cohen, G. (2003), A course in modern analysis and its applications, Australian Mathematical Society lecture series, vol. 17, Cambridge University Press, Port Melbourne, Australia

Colorni, A.; Dorigo, M.; Maffoli, F.; Maniezzo, V.; Righini, G. \& Trubian, M. (1996). Heuristics from nature for hard combinatorial optimisation problems. International Transactions in Operational Research, 3, 1, 1-21

Cunha, M.C., \& Ribeiro, L. (2004). Tabu search algorithms for water network optimization. European Journal of Operational Research, 157, 746-758

Cuhna, M.C. \& Sousa, J. (1999). Water distribution network design optimization: Simulated annealing approach. Journal of Water Resources Planning and Management, ASCE, 125, 4, 215-221

Dandy, G.C.; Simpson, A.R. \& Murphy, L.J. (1996). An improved genetic algorithm for pipe network optimisation. Water Resources Research, 32, 2, 449-458

Deb, K. (2001). Multi-objective optimization using evolutionary algorithms, Wiley, Chichester, U.K.

Dorigo, M.; Bonabeau E., \& Theraulaz, G. (2000). Ant algorithms and stigmergy. Future Generation Computer Systems, 16, 851-871

Dorigo, M.; Di Caro, G. \& Gambardella, L.M. (1999). Ant algorithms for discrete optimisation. Artificial Life, 5, 2, 137-172

Dorigo, M. \& Gambardella, L.M. (1997). Ant colony system: A cooperative learning approach to TSP. IEEE Transactions on Evolutionary Computation, 1, 1, 53-66

Dorigo, M.; Maniezzo, V. \& Colorni, A. (1996). The ant system: Optimisation by a colony of cooperating agents. IEEE Transactions on Systems, Man, and Cybernetics - Part B, 261 , 29-41

Eusuff, M.M. \& Lansey, K.E. (2003). Optimisation of water distribution network design using the shuffled frog leaping algorithm. Journal of Water Resources Planning and Management, ASCE, 129, 3, 210-225 
Fujiwara, O. \& Khang, D.B. (1990). A two phase decomposition method for optimal design of looped water distribution networks. Water Resources Research, 26, 4, 539-549

Gessler, J. (1982). Optimisation of pipe networks. Proceedings of International Symposium on Urban Hydrology, Hydraulics and Sediment Control. University of Kentucky, Lexington, 165-171

Gessler, J. (1985). Pipe network optimisation by enumeration. Computer Applications for Water Resources, ASCE, Buffalo, NY, 76, 579-581

Gutjahr, W.J. (2002). ACO algorithms with guaranteed convergence to the optimal solution. Information Processing Letters, 82, 145-153

Maier, H.R.; Simpson, A.R., Zecchin, A.C.; Foong, W.K.; Phang, K.Y.; Seah, H.Y. \& Tan, C.L. (2003). Ant colony optimisation for the design of water distribution systems. Journal of Water Resources Planning and Management, ASCE, 129, 3, 200-209

Rossman (2000). EPANET 2 Users Manual. National Risk Management Research Laboratory Office of Research and Development U.S. Environmental Protection Agency. Cincinnati, Ohio, USA.

Savic, D.A. \& Walters, G.A. (1997). Genetic algorithms for least-cost design of water distribution networks. Journal of Water Resources Planning and Management, ASCE, $123,2,67-77$

Schaake, J. \& Lai, D. (1969). Linear programming and dynamic programming applications to water distribution network design. Research Report No. 116, Department of Civil Engineering, Massachusetts Institute of Technology

Simpson, A.R.; Murphy, L.J. \& Dandy, G.C. (1994). Genetic algorithms compared to other techniques for pipe optimization. Journal of Water Resources Planning and Management, ASCE, 120, 4, 423-443

Simpson, A.R., \& Goldberg, D.E. (1994). Pipeline optimization via genetic algorithms: From theory to practice. Proc. $2^{\text {nd }}$ Int. Conf. On Pipeline Systems, Edinburgh, Scotland, May, 309-320

Sousa, J. \& Cunha, M.C. (1999), On the quality of simulated annealing for water network optimization problems, In: Water Industry Systems: Modelling and Optimisation Applications, Vol. 2, Savic, D., Walters, G. (Eds.), 333-345, Research Studies Press Ltd.

Streeter, V. L.; Wylie, E. B. \& Bedford, K. W. (1997), Fluid mechanics, 9th ed.,WCB/McGraw Hill, Boston, Mass.

Stützle, T. \& Hoos, H.H. (2000). MAX-MIN Ant System. Future Generation Computer Systems, $16,889-914$

Wu, Z.Y.; Boulos, P.F.; Orr, C.H. \& Ro, J.J. (2001). Using genetic algorithms to rehabilitate distribution systems. Journal for American Water Works Association, November 2001, 74-85

Zecchin, A.C.; Simpson, A.R.; Maier H.R., \& Nixon, J.B. (2005). Parametric study for an ant algorithm applied to water distribution system optimization. IEEE Transactions on Evolutionary Computation, 9, 2, 175-191

Zecchin, A.C.; Maier, H.R.; Simpson, A.R.; Leonard, M.; Roberts, A.J., \& Berrisford, M.J. (2006). Application of two ant colony optimisation algorithms to water distribution system optimisation. Mathematical and Computer Modelling, 44, 451-468

Zecchin, A.C.; Maier, H.R.; Simpson, A.R.; Leonard, M., \& Nixon, J.B. (2007). Ant Colony Optimization Applied to Water Distribution System Design: Comparative Study of Five Algorithms. ASCE Journal of Water Resources, Planning \& Management, 133, 1, $84-92$ 


\section{Swarm Intelligence,} Focus on Ant and Particle Swarm Optimization

\section{IMTECH}

\section{Swarm Intelligence, Focus on Ant and Particle Swarm Optimization \\ Edited by FelixT.S.Chan and Manoj KumarTiwari}

ISBN 978-3-902613-09-7

Hard cover, 532 pages

Publisher I-Tech Education and Publishing

Published online 01, December, 2007

Published in print edition December, 2007

In the era globalisation the emerging technologies are governing engineering industries to a multifaceted state. The escalating complexity has demanded researchers to find the possible ways of easing the solution of the problems. This has motivated the researchers to grasp ideas from the nature and implant it in the engineering sciences. This way of thinking led to emergence of many biologically inspired algorithms that have proven to be efficient in handling the computationally complex problems with competence such as Genetic Algorithm (GA), Ant Colony Optimization (ACO), Particle Swarm Optimization (PSO), etc. Motivated by the capability of the biologically inspired algorithms the present book on "Swarm Intelligence: Focus on Ant and Particle Swarm Optimization" aims to present recent developments and applications concerning optimization with swarm intelligence techniques. The papers selected for this book comprise a cross-section of topics that reflect a variety of perspectives and disciplinary backgrounds. In addition to the introduction of new concepts of swarm intelligence, this book also presented some selected representative case studies covering power plant maintenance scheduling; geotechnical engineering; design and machining tolerances; layout problems; manufacturing process plan; job-shop scheduling; structural design; environmental dispatching problems; wireless communication; water distribution systems; multi-plant supply chain; fault diagnosis of airplane engines; and process scheduling. I believe these 27 chapters presented in this book adequately reflect these topics.

\section{How to reference}

In order to correctly reference this scholarly work, feel free to copy and paste the following:

Aaron C. Zecchin, Holger R. Maier and Angus R. Simpson (2007). Case Study Based Convergence Behaviour Analysis of ACO Applied to Optimal Design of Water Distribution Systems, Swarm Intelligence, Focus on Ant and Particle Swarm Optimization, FelixT.S.Chan and Manoj KumarTiwari (Ed.), ISBN: 978-3-902613-09-7, InTech, Available from:

http://www.intechopen.com/books/swarm_intelligence_focus_on_ant_and_particle_swarm_optimization/case_ study_based_convergence_behaviour_analysis_of_aco_applied_to_optimal_design_of_water_distributi

\section{INTECH}

open science | open minds

\author{
InTech Europe \\ University Campus STeP Ri \\ Slavka Krautzeka 83/A \\ 51000 Rijeka, Croatia \\ Phone: +385 (51) 770447 \\ Fax: +385 (51) 686166
}

\author{
InTech China \\ Unit 405, Office Block, Hotel Equatorial Shanghai \\ No.65, Yan An Road (West), Shanghai, 200040, China \\ 中国上海市延安西路65号上海国际贵都大饭店办公楼405单元 \\ Phone: +86-21-62489820 \\ Fax: $+86-21-62489821$
}


www.intechopen.com 
(C) 2007 The Author(s). Licensee IntechOpen. This chapter is distributed under the terms of the Creative Commons Attribution-NonCommercial-ShareAlike-3.0 License, which permits use, distribution and reproduction for non-commercial purposes, provided the original is properly cited and derivative works building on this content are distributed under the same license. 